\title{
EMPRENDIMIENTOS TEMPRANOS DE INMIGRANTES JUDÍOS en VAlParaíso Y Viña del Mar, 1920-1944
}

\begin{abstract}
RESUMEN
El artículo caracteriza los primeros emprendimientos desarrollados por inmigrantes judíos entre 1920 y 1944 en la ciudad de Valparaíso y Viña del Mar, a partir de una metodología cuantitativa aplicada a sesenta y cinco empresarios y sesenta y siete establecimientos. El trabajo revela que la mayor parte de sus establecimientos no se concentraron en términos de propiedad, se localizaron esencialmente en Valparaíso a lo largo de las arterias comerciales que comunicaban el plan de la ciudad y fueron predominantemente comerciales -aunque hubo algunos industriales- y encadenaron fabricación y venta a través de pequeños talleres-tienda. Además, muestra que estos empresarios fueron askenazíes -si bien hubo una proporción no insignificante de sefardíes- que arribaron al país en la década de 1920, casi sin estudios universitarios y que tardaron al menos quince años en prosperar. Por último, que su acción fue el basamento material de la primera red de organizaciones judías en Valparaíso y Viña del Mar.
\end{abstract}

Palabras claves: Valparaíso, Viña del Mar, siglo xx, inmigrantes judíos, actividad económica.

\section{Abstract}

This article characterizes the first undertakings developed by Jewish immigrants between 1920 and 1944 in the city of Valparaiso and Viña del Mar, starting from a quantitative methodology applied to 65 business owners and 67 establishments. This work reveals that the majority of their establishments were not concentrated in terms of property, they were found mainly in Valparaíso throughout the commercial arteries that connected the lower plane of the city and were predominantly commercial -although some were industrial- and linked production and sales through small workshops-stores. Additionally, it shows that these entrepreneurs were Ashkenazi Jews- although there was a not insignificant proportion of Sephardic Jews- that arrived to the country during the 1920 s, almost all without university education and took at least 15 years to become successful. Lastly, their actions served as the material basis for the first network of Jewish organizations in Valparaíso and Viña del Mar.

\footnotetext{
* Dr. Historia Económica, académico de la Universidad de Chile. Correo electrónico: mmatus@uchile.cl

${ }^{1}$ Artículo elaborado en el marco del proyecto FONDECYT No 1160374 de la Comisión Nacional de Investigación Científica y Tecnológica de Chile (CONICYT).
} 
Keywords: Valparaíso, Viña del Mar, twentieth-century, Jewish immigrants, economic activity.

Recibido: Octubre 2017.

Aceptado: Marzo 2018.

\section{INTRODUCCIÓN}

Según los Censos de la República de Chile, hasta 1907 en la zona comprendida por la actual V Región de Valparaíso residían solo veinte personas que se declaraban de religión judía, lo que dejaba a esta región con una muy reducida presencia judía, incluso inferior a la de la Región de Magallanes (veinticuatro personas) y apenas superior a la de la Región de la Araucanía (catorce personas). Pero entre los censos de 1920 y 1952 ese número ascendió desde 261 a 843 personas, lo que permitió que la Región de Valparaíso se convirtiera en la segunda región con mayor presencia judía luego de la Región Metropolitana, dejando muy atrás el aporte de otras regiones, como la VIII del Biobío (261), IX De la Araucanía (291), X De Los Lagos (223) y IV Coquimbo (88 personas) ${ }^{2}$. En consecuencia, el arribo y localización de una inmigración judía importante en la Región de Valparaíso solo comenzó a ser significativa entre 1920 y 1952, beneficiando sustancialmente a esa ciudad. Sin embargo, muy poco se ha dicho sobre las actividades de este grupo de inmigrantes, ya que la atención se ha concentrado esencialmente en la ciudad de Santiago, y en menor medida, Temuco ${ }^{3}$. El propósito de este artículo es, precisamente, identificar los rasgos que caracterizaron las principales actividades económicas desarrolladas por estos inmigrantes judíos de primera generación en Valparaíso y Viña del Mar, poniendo como límite el año de 1944, pues ese año se publicó una obra referencial como catastro de la comunidad judía en Chile y que fue una de las fuentes de este estudio ${ }^{4}$.

Este trabajo reunió en tres ámbitos la hipótesis que parecían plausibles al inicio de esta investigación y que fueron sometidas a contraste: 1) En el ámbito correspondiente

\footnotetext{
${ }^{2}$ Mario Matus, Tradición y adaptación, vivencia de los sefaradies en Chile, Santiago, Universidad de Chile, Departamento de Ciencias Históricas y Comunidad Israelita Sefaradí de Chile, 1993, p. 73. Debe recordarse que la única forma de averiguar la pertenencia judía en los censos es a través de la adscripción religiosa declarada, ya que en la categoría nacionalidad aparecen como ciudadanos procedentes de múltiples países. A su vez, la adscripción de tipo religiosa posibilita dos distorsiones inevitables. Por un lado, chilenos simpatizantes del judaísmo podrían engrosar la contabilidad; por otro, judíos no religiosos podrían no ser registrados. Afortunadamente, la mayor parte de los censados solían declararse como judíos a pesar de no ser religiosos, en tanto interpretaban la pregunta en un sentido más amplio que el meramente religioso. Del mismo modo, las cifras son bastante reducidas, por lo que un eventual abultamiento debe haber sido insignificante. En suma, los valores pueden tomarse como cercanos a la realidad.

${ }^{3}$ Para Santiago puede consultarse la obra de Moisés Senderey, Historia de la colectividad israelita de Chile, Santiago, Editorial "Dos Ydische Wort", 1956, pp. 216-222. Para Santiago y Temuco es de utilidad la obra de Moshé Nes-El, Historia de la comunidad israelita sefaradí de Chile, Santiago, Nascimento, 1984, pp. 243-261.

${ }^{4}$ Luis Aguirre, Álbum gráfico y biográfico de los Israelitas en Chile, Valparaíso, Ed. El Mercurio de Valparaíso, 1944.
} 
a sus establecimientos, se planteó que estuvieron poco concentrados en términos de propiedad, que se localizaron en una zona acotada de la ciudad y que fueron abrumadoramente comerciales, con nula participación industrial; 2) Respecto a estos empresarios, se aventuró que en su mayor parte eran de origen askenazí, que tenían estudios universitarios incompletos o terminados y que prosperaron relativamente rápido, en mucho menos de una década y 3) En relación con su impacto, pareció que estos emprendimientos constituyeron el fundamento material de la red de organizaciones comunitarias que nació en Valparaíso y que luego se extendió a Viña del Mar.

\section{FUENTES Y METODOLOGÍA}

Para poner a prueba estas intuiciones iniciales se construyó una base de datos en Excel $^{5}$ conformada por veintitrés variables: 1) Primer apellido, 2) Primer nombre propio, 3) Ads-cripción étnico-cultural (sefardí-askenazí), 4) Año de llegada a Chile, 5) Generación, 6) Fecha de nacimiento, 7) Fecha de defunción, 8) Sepultación en mausoleo judío del cementerio de Playa Ancha, 9) Sepultación en mausoleo judío de cementerio de El Belloto, 10) Constancia de estudios universitarios, 11) Nombre de establecimiento, 12) Rubro de actividad económica, 13) Primer registro de establecimiento, 14) Último registro de establecimiento hasta 1944, 15) Eventual sucursal, 16) Dirección 1 de establecimiento, 17) Última dirección hacia 1944, 18) Teléfono de establecimiento, 19) Participación comunitaria 1,20) Participación comunitaria 2, 21) Participación comunitaria 3, 22) Eventual donante para edificio Sociedad Unión Israelita de Valparaíso y 23) Eventual donante de Keren Hayesod ${ }^{6}$. Esta base de datos incorporó numerosas fuentes primarias $^{7}$ y algunas obras secundarias ${ }^{8}$. Debo los datos de los mausoleos judíos del cementerio de Playa Ancha y en el cementerio del Belloto a la generosidad de Baldomero Estrada.

El listado de sesenta y cinco empresarios y sesenta y siere establecimientos identificados aparece en el cuadro 1. No coinciden exactamente porque, en ocasiones, una persona tenía más de un establecimiento y, en otras, el establecimiento era compartido (por ejemplo, con un hermano), casos señalizados mediante el uso de comillas (“ ”).

\footnotetext{
${ }^{5}$ Agradezco a Ignacio Pérez Eyzaguirre su valiosa contribución en la construcción de esta base de datos, como a Uziel González su importante trabajo de digitalización de las publicaciones de organizaciones judías chilenas a lo largo del siglo xx.

${ }^{6}$ Institución fundada en 1920, como brazo recaudador de fondos del Movimiento Sionista.

${ }^{7}$ Entre las que destaca El Mercurio de Valparaíso entre 1901 y 1930, y las revistas comunitarias Renacimiento (1919), Nosotros (1926-1930), Alma Hebrea (1931-1935), Boletín de la Federación Sionista de Chile (1932-1934), Unzer Lebn (1936-1937), El mundo (1937-1939), La voz de la WIZO (1942-1948), Boletín de informaciones del círculo israelita (1940-1941), Jalutz (1944-1945), Anuario de la Federación Sionista de Chile (1945-1947) y Revista de la JIS, Juventud Israelita Sefaradi (1945). A ellas se agregó la obra de la Sociedad de Fomento Fabril, Rol industrial de Chile, Santiago, Impresores “El Imparcial”, 1960.

${ }^{8}$ A estas fuentes se agregó las obras de Juan Romero Córdova, Testimonios de inmigrantes judios y su aporte en el Valparaíso del 1900, Valparaíso, Ediciones Facultad de Humanidades, Universidad de Playa Ancha, 2002; Moshé Nes-El, Estudios sobre el Judaismo Chileno, Jerusalén, Ediciones Revista de Oriente y Occidente, 2009 y Eduardo Weinstein et al., Sueños de Libertad, Legado de los primeros inmigrantes judíos llegados a Valparaíso, Santiago, Editorial 3iG, 2016.
} 
Se seleccionó a los establecimientos que cumplieron los siguientes requisitos: 1) Estar localizados en Valparaíso o Viña del Mar entre 1920 y 1944, 2) Ser propiedad de una persona de primera generación, es decir, de inmigrantes directos y 3) Aportar datos mínimos (rubro y dirección al menos; y ojalá el nombre). En cuanto a los propios empresarios, se seleccionó aquellos que al menos aportaban apellido y pertenencia étnica -askenazí o sefardí ${ }^{9}$, y en lo posible, el año aproximado de su llegada. En segundo término, se procuró que en la mayoría de los casos se supiera si en algún momento del periodo 1920-1944 fueron donantes, socios o dirigentes de instituciones comunitarias judías, y el lugar donde fueron sepultados.

\section{CUADRO 1}

Listado de empresarios y establecimientos judios

(Valparaíso-Viña del Mar, 1920-1944)

\begin{tabular}{|c|c|c|c|}
\hline $\mathrm{N}^{\circ}$ & Empresari@ & $\mathrm{N}^{\circ}$ & Establecimiento y primer registro \\
\hline 1 & L. Abramovich & 1 & Sastrería Richmond (1928) \\
\hline \multirow[t]{2}{*}{2} & Moisés Abramson & 2 & Sastrería La Elegancia (1928) \\
\hline & “ $\quad "$ & 3 & Sastrería femenina Marymor (1927) \\
\hline 3 & Marcos Agosin & 4 & Sastrería Agosin (1933) suc. Quillota (1944) \\
\hline 4 & Victor Alaluf & 5 & Tienda de lujo La Chatelett (1944) \\
\hline 5 & Salvador Albajari & 6 & Frutos del país (1933) \\
\hline 6 & Moreno Esquenazi & & “ $\quad "$ \\
\hline 7 & Alberto Avayú & 7 & Tienda Las dos californias (1923) \\
\hline 8 & Isaac Avayú & 8 & Importac. y confección arts. lana y algodón (1937) \\
\hline 9 & Jacobo Blinder & 9 & Fca. de muebles La Sudamericana (1937) \\
\hline 10 & Samuel Bronfman & 10 & Peletería Boston (1927) \\
\hline 11 & Budnik & 11 & Residencial Budnik (Viña del Mar), (1934) \\
\hline 12 & Bernardo Burstein & 12 & Distribución de soya y jabón (1937) \\
\hline 13 & Salomón Cohen & 13 & Fábrica de camisas Cohen (1925 y 1937) \\
\hline 14 & Bernardo Dinovitzer & 14 & Casa Dinovitzer (1928) \\
\hline 15 & Domb & 15 & Domb y Cía. (1937) \\
\hline 16 & Alfredo Ephrosi & 16 & Fca. de vidrios-cristales. Ind. vidrios Ltda. (1940) \\
\hline 17 & Ricardo Freudenberg & & “ $\quad "$ \\
\hline 18 & José Finkelstein & 17 & Sastrería High Life (1927) \\
\hline \multirow[t]{2}{*}{19} & Isidoro Froimovich & 18 & Fábrica de bebidas gaseosas (1925) \\
\hline & “ $"$ & 19 & Bodega frutos del país (1939) \\
\hline 20 & Adolfo Gomberoff & 20 & Fábrica de muebles La Argentina (1939) \\
\hline \multirow[t]{2}{*}{21} & Samuel Grimblatt & 21 & Sastrería Bristol (1929) \\
\hline & “ $\quad "$ & 22 & Sastrería Esmeralda (1944) \\
\hline 22 & Benjamín Grossman & 23 & Peletería y tintorería Las Novedades (1936) \\
\hline 23 & Juan Guiloff & 24 & Sastrería La Fama (1927) \\
\hline 24 & Gun & 25 & Gun y Cía. frutos del país y abarrotes (1944) \\
\hline 25 & M. Heller & 26 & Tintorería Los mil colores (1927) \\
\hline
\end{tabular}

\footnotetext{
${ }^{9}$ En este caso, se ha usado el adjetivo 'étnico' para enfatizar que no se trata de una adscripción religiosa, sino, más bien, de vínculos de parentesco, como trayectorias culturales e históricas distintas.
} 


\begin{tabular}{|c|c|c|c|}
\hline $\mathrm{N}^{\circ}$ & Empresari@ & $\mathrm{N}^{\circ}$ & Establecimiento y primer registro \\
\hline 26 & Emmanuel Hirnheimer & 27 & Artefactos sanitarios La Europea (1940?) \\
\hline 27 & Elías Horovitz & 28 & Peletería Londres (1920) \\
\hline 28 & Cecilia Jajam & 29 & Sombrerería La Victoria (1920) \\
\hline 29 & Miguel Jusid & 30 & Importadora de telas Miguel Jusid (1927) \\
\hline 30 & Aron Kerner & 31 & Peletería y tienda "Kerner" (1936) \\
\hline 31 & Máximo Kerner & & “ $\quad$ ", sucursal Viña del Mar (1936) \\
\hline 32 & Marcos Kleiman & 32 & Pensión Kleiman (1936) \\
\hline \multirow[t]{2}{*}{33} & Bernardo Kramarenko & 33 & Peletería Condell (1928) \\
\hline & “ $\quad "$ & 34 & Cambio de monedas (1936) \\
\hline 34 & Sigfredo Kromacher & 35 & Productos de cuero Kromacher (1942) \\
\hline 35 & Ernesto Kromacher & & “ $\quad "$ \\
\hline 36 & Isaac Kuperman & 36 & Bodega Kuperman (1939) \\
\hline 37 & Carlos Kuschnier & 37 & Sastrería Kuschnier (1935) \\
\hline 38 & David Lanis & 38 & Maison Lanis, venta de arts. de lujo (1928) \\
\hline 39 & Gregorio Levinson & 39 & Peletería Francia (1927) \\
\hline 40 & Jaime Levy & 40 & Frutería Levy (1935) \\
\hline \multirow[t]{2}{*}{41} & Victor Lifschitz & 41 & Fca. de carteras Paris, Condell (1927) suc. Stgo. (1936). \\
\hline & “ $\quad "$ & 42 & Fca. de carteras Paris, Pedro Montt (1927) \\
\hline \multirow[t]{2}{*}{42} & León Link & 43 & Mueblerías Colón (1941) \\
\hline & “ $\quad "$ & 44 & Mueblería Link (1944) \\
\hline 43 & Clara Litvak de Hirsch & 45 & Farmacia Nueva París (1941) \\
\hline 44 & Jaime Mandel & 46 & Lenc.Tikwa/ imp. Sylom (1926) suc Stgo. (1944) \\
\hline 45 & José Medvinsky & 47 & Pelet. Sud-Americana (Stgo. 1919), Valpo, 1926 \\
\hline 46 & Alberto Messina & 48 & Paquetería La Central (1935) \\
\hline \multirow[t]{2}{*}{47} & Samuel Michaely & 49 & Casa-Multitienda Michaely (1941) \\
\hline & “ $\quad "$ & 50 & Tienda Samy (1944) \\
\hline 48 & Marcos Nahum & 51 & Paquetería El Porvenir (1935) \\
\hline 49 & Paulsen & 52 & Paulsen y Cía. Distrib. de máquinas de escribir (1937) \\
\hline 50 & José Roscinsky & 53 & Mueblería Temuco (1941) \\
\hline \multirow[t]{2}{*}{51} & Gregorio Roizblatt & 54 & Dr. consulta médica (1935) \\
\hline & “ & 55 & Peletería y sombrerería Los Leones (1941) \\
\hline 52 & Felipe Schapira & 56 & Peletería El zorro gris (1927) \\
\hline 53 & Mauricio Schwartstein & 57 & Muebles Shwartstein (1944) \\
\hline \multirow[t]{2}{*}{54} & Isaac Smirnoff & 58 & Gran peletería Yarcho, Condell (1925) \\
\hline & “ & 59 & Peletería-sombrerería El León, Victoria (1925) \\
\hline 55 & Max Strauss & 60 & Hospedería Strauss (Viña del Mar) (1944) \\
\hline 56 & Alejandro Strauss & & “ $"$ \\
\hline 57 & Alberto Sverdlov & 61 & Mueblería Universal (1944) \\
\hline 58 & Alberto Ventura & 62 & Tienda Europa (1939) \\
\hline 59 & Isaac Ventura & & “ $\quad "$ \\
\hline 60 & Mauricio Volosky & 63 & Joyería-relojería “El palacio de los novios”, 1941 \\
\hline 61 & José Weinstein & 64 & Mueblería y Tapicería “Londres” (1927) \\
\hline 62 & Julio Weinstein & & “ \\
\hline 63 & Marcos Weinstein & 65 & Dr. Consulta médica (1941) \\
\hline 64 & Weiss & 66 & Casa Weiss, casa de cambio de monedas (1936) \\
\hline 65 & Salomón Zeldis & 67 & Joyería-relojería Election, palacio de relojes, (1944) \\
\hline
\end{tabular}


Los datos de otros once empresarios y diez establecimientos, que se localizaban en otras ciudades, pertenecían a otro periodo, eran de segunda generación, o no aportaban los datos esenciales aparecen en el cuadro 2 (datos excluidos del trabajo).

CuAdro 2

Listado de once empresarios judios y diez establecimientos descartados

\begin{tabular}{|c|c|c|c|c|}
\hline $\mathrm{N}^{\circ}$ & Empresari@ & $\mathrm{N}^{\circ}$ & Establecimiento & Datos ausentes \\
\hline 1 & Victor Alaluf & 1 & Industria del cuero & Dirección \\
\hline 2 & Julián Arendt & 2 & $1^{\mathrm{a}}$ línea de buses entre Valpo. y Quilpué & Fecha y dirección \\
\hline 3 & Horst Arendt & 3 & Taller de relojería & Fecha y dirección \\
\hline 4 & Alberto e Isaac Avayú & 4 & Fábrica de ropa hecha & Fecha y dirección \\
\hline 5 & Julio Berstein & 5 & Refinería de Azúcar de Viña del Mar & Todo, fuera período \\
\hline 6 & Salomón Cohen & 6 & Negocio no determinado (1925) & Rubro y dirección \\
\hline 7 & David Grossman & 7 & Sastrería Confecciones Grossman & $2^{\mathrm{a}}$ generación \\
\hline 8 & Salomón Grossman & & 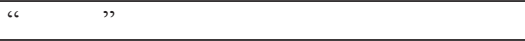 & $2^{\mathrm{a}}$ generación \\
\hline 9 & Alberto Sacks & 8 & Frutos del país Cía. Central-Bus (Valpo-Viña) & Fecha y dirección \\
\hline 10 & Rodolfo Staub & 9 & Mueblería Staub & Todo \\
\hline 11 & Abraham Wortzman & 10 & Exportación de productos agrícolas & Los Andes \\
\hline
\end{tabular}

De tal modo, el trabajo consistió en analizar los datos de los empresarios y sus establecimientos a lo largo de tres ejes: 1) Sus sesenta y siete establecimientos, en cuanto a grado de concentración en términos de propiedad, sector económico y rubros de actividad predominantes, su escala de operaciones y su localización dentro de Valparaíso y Viña del Mar; 2) Los propios sesenta y cinco empresarios judíos, a través de su origen étnico (askenazí o sefardí), si tenían estudios universitarios (completos e incompletos), el tiempo que demoraron en instalar un segundo establecimiento en Valparaíso y Viña del Mar o en otras ciudades y 3) El grado de vinculación o compromiso comunitario que ellos tenían, a través de donaciones a proyectos o a causas vinculadas a la colectividad, de su participación como dirigentes en organizaciones judías y de su sepultación con ritual religioso judío.

Se debe tomar en cuenta que todos los datos de los establecimientos estuvieron disponibles, de modo que el universo siempre fue sesenta y siete. Pero de los sesenta y cinco empresarios, uno de ellos no aportó el dato de año de llegada. Por otro lado, no se contó con la información para un número de ellos en lo relativo a donaciones, cargos como socios o dirigentes y el lugar donde fueron sepultados, por lo que, en esos casos, se introdujo la expresión "Sin información (S/I) o no donantes, S/I o no socios o sin cargos y S/I o no sepultados con rito judío". Así y todo, los resultados fueron lo suficientemente contundentes como para extraer de ellos constataciones relativamente sólidas.

\section{RASGOS PRIMORDIALES DE SESENTA Y SIETE ESTABLECIMIENTOS JUDÍOS DEL PERIOdo 1920-1944, en VALPARAíso y ViÑa del Mar}

En primer término, se midió el relativo grado de concentración de los establecimientos pertenecientes a los sesenta y cinco empresarios identificados. En este caso, el gráfico 1 registra que la mayoría de estos emprendedores eran relativamente modestos y que la 
propiedad estaba bastante distribuida hacia 1944, ya que cincuenta y dos de ellos (80\%) eran propietarios de un único establecimiento hacia 1944 , mientras que doce $(18,5 \%)$ eran propietarios de dos establecimientos y solo uno $(1,5 \%)$ logró tener tres establecimientos ${ }^{10}$

\section{GRÁFICO 1}

Grado de concentración en establecimientos (en \%)

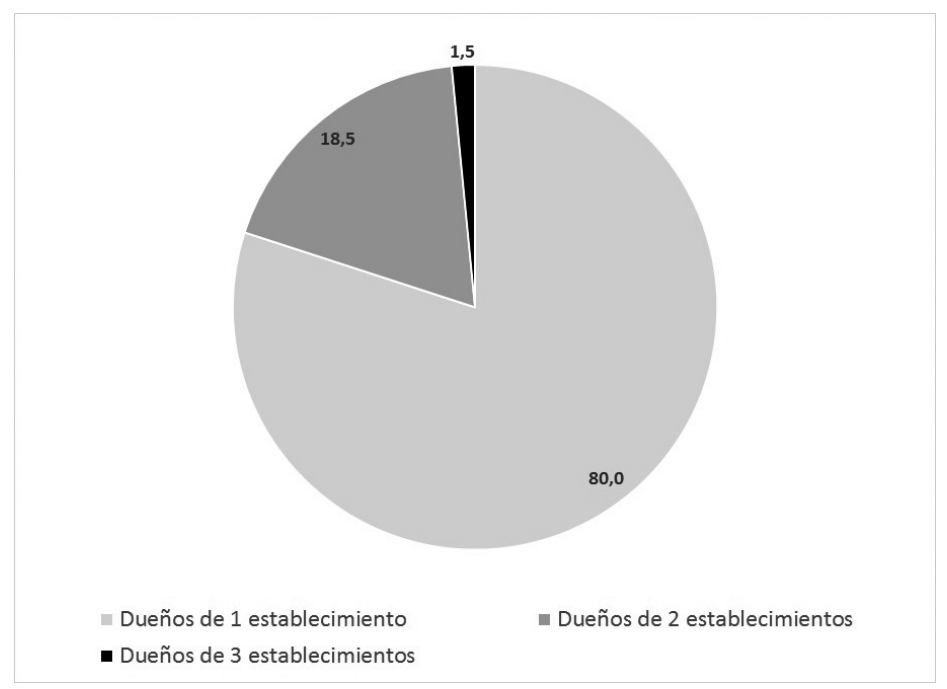

Al indagar en los casos en que un empresario tiene más de un establecimiento, se advierte que en muchas ocasiones se expresaba en sucursales de una primera tienda o taller, ya fuera en Valparaíso y Viña del Mar o en otras ciudades cercanas, lo que implicaba simplemente replicar un modelo de negocios conocido y que ya marchaba de forma correcta. Aquí se pudieron situar las dos sastrerías de Moisés Abramson, las dos sastrerías de Marcos Agosin, las dos sastrerías de Samuel Grimblatt, las dos peleterías de los hermanos Kerner, las tres fábricas de carteras de Victor Lifschitz, las dos mueblerías de León Link, las dos peleterías de José Medvinsky, las dos tiendas de Samuel Michaely y las dos peleterías de Isaac Smirnoff. En otras situaciones, en cambio, tal vez fue consecuencia de formas de integración vertical, que animaban a reunir en un mismo espacio dos actividades distintas. Es lo que probablemente ocurrió con la fábrica de bebidas gaseosas y la bodega de frutos del país de Isidoro Froimovich, situadas en la misma dirección (Cochrane 121), quizá para aprovechar las frutas de la bodega como insumos en la fabricación de bebidas gaseosas. Otro tipo de integración vertical debe haber sido el caso de la importadora y lencería que Jaime Mandel situó en Salvador Donoso 250. En otros casos, una misma dirección posibilitó costes reducidos para actividades muy poco integradas (como la peletería y casa de cambio de monedas que Bernardo Kramarenko

\footnotetext{
${ }^{10}$ La sumatoria no coincide con los sesenta y siete establecimientos, ya que para este cálculo no solo se consideraron los establecimientos en Valparaíso y Viña del Mar sino que, también, las sucursales en otras ciudades (Santiago y Quillota).
} 
situó en José Tomás Ramos 140) o como la consulta médica y peletería que Gregorio Roizblatt situó en dos direcciones contiguas (Victoria 2364 y 2364).

En cuanto al sector económico al que pertenecían estos sesenta y siete establecimientos, sesenta y cuatro de ellos $(95,5 \%)$ pertenecían al sector terciario, a través de variados servicios, mientras que solo tres $(4,5 \%)$ eran propiamente industriales (fábrica de vidrios y cristales de Alfredo Ephrosi, fábrica de bebidas gaseosas de Isidoro Froimovich y fábrica de Camisas de Salomón Cohen) $)^{11}$.

Pero fuera de este claro predominio de las actividades terciarias, el gráfico 2 evidencia que estas fueron mucho más variadas de lo que se imaginaba, distinguiéndose un primer grupo de gran presencia constituido por peleterías y fábricas de artículos de cuero (14), sastrerías (9), mueblerías (8) y venta minoritaria de ropa y lencerías (6). En números mucho más reducidos se situaban los almacenes de frutos del país y abarrotes (4) y hospederías y residenciales (3). Mucho más abajo, aparecen con dos establecimientos las paqueterías, importadoras de ropa, venta de artículos de lujo, relojerías y joyerías, casas de cambio y consultas médicas, dejando con un establecimiento las sombrererías, la venta de artefactos sanitarios, la distribución de máquinas de escribir, las farmacias, las multitiendas, las compraventas de fierro y metales, las distribuidoras de aceite y jabón y las tintorerías.

GrÁFICO 2

Establecimientos judios en Valparaíso y Viña del Mar, 1920-1944

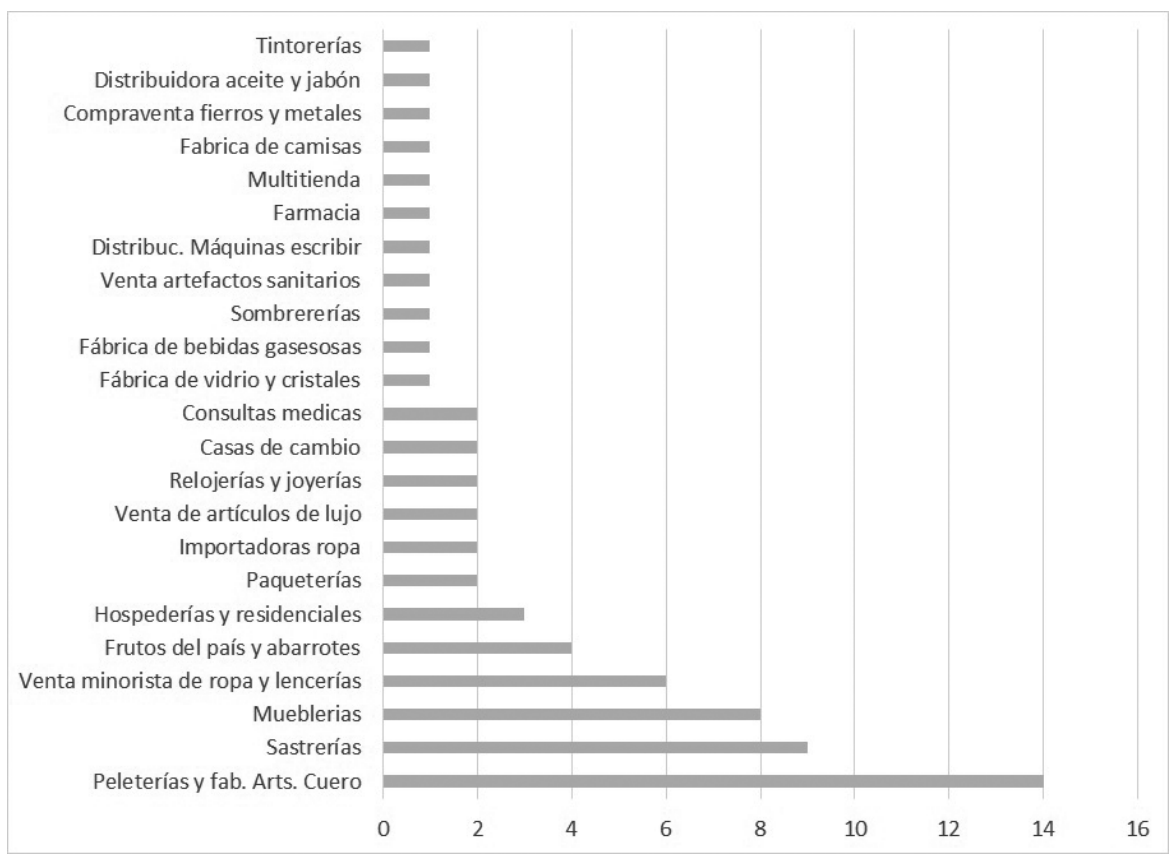

\footnotetext{
${ }^{11}$ Luis Aguirre, señala que en el primer caso se trataba de todo un edificio que ocupaba numerosas máquinas y operarios, en el segundo caso, que enviaba sus productos al norte del país y que sus trabajadores disfrutaban de gran estabilidad y, en el tercer caso, que a pesar de ser numerosos sus trabajadores, no se daban conflictos laborales. Quizá hubiera integración entre las dos primeras. Cfr. Aguirre, op. cit., pp. 43, 28 y 52, respectivamente.
} 
En relación con la escala de sus operaciones, los sesenta y siete establecimientos fueron clasificados en las siguientes categorías: 1) Pequeños talleres, 2) Grandes talleres, 3) Establecimientos industriales, 4) Talleres y comercio minorista, 5) Comercio minorista, 6) Comercio mayorista y 7) Servicios profesionales y hospederías. El gráfico 3 muestra esta distribución.

Este gráfico puede leerse de modo similar a una pirámide de estructura por edades en cierto año, en la que la instantánea temporal no solo captura los grupos de edad sino, también, sugiere los desplazamientos verticales entre ellos desde los grupos inferiores hacia arriba. En ese sentido, corrobora que la escala de operaciones de los establecimientos estuvo muy vinculada a mecanismos de eslabonamiento productivo -cuyos trazos ya se advirtieron en el apartado sobre grado de concentración en la propiedad. La lógica era de acumulación familiar y probablemente funcionaba así. En un primer momento, que no aparece en el gráfico, pero estaría debajo de los grupos inferiores, muchos de estos emprendedores -como está bastante documentado en numerosos testimonios- se dedicaban a la venta minorista ambulante, a lo largo de los cerros de Valparaíso, varias veces auxiliados por mulas o caballos que trasladaban los enseres. La venta era a plazos y en cuotas, donde, por lo general, la primera cancelaba un porcentaje importante del costo del artículo. Excepcionalmente, si alguno contaba con formación universitaria completa, podía establecerse como profesional independiente.

GRÁFICO 3

Escala de operaciones de establecimientos judíos en Valparaíso y Viña del Mar, 1920-1944

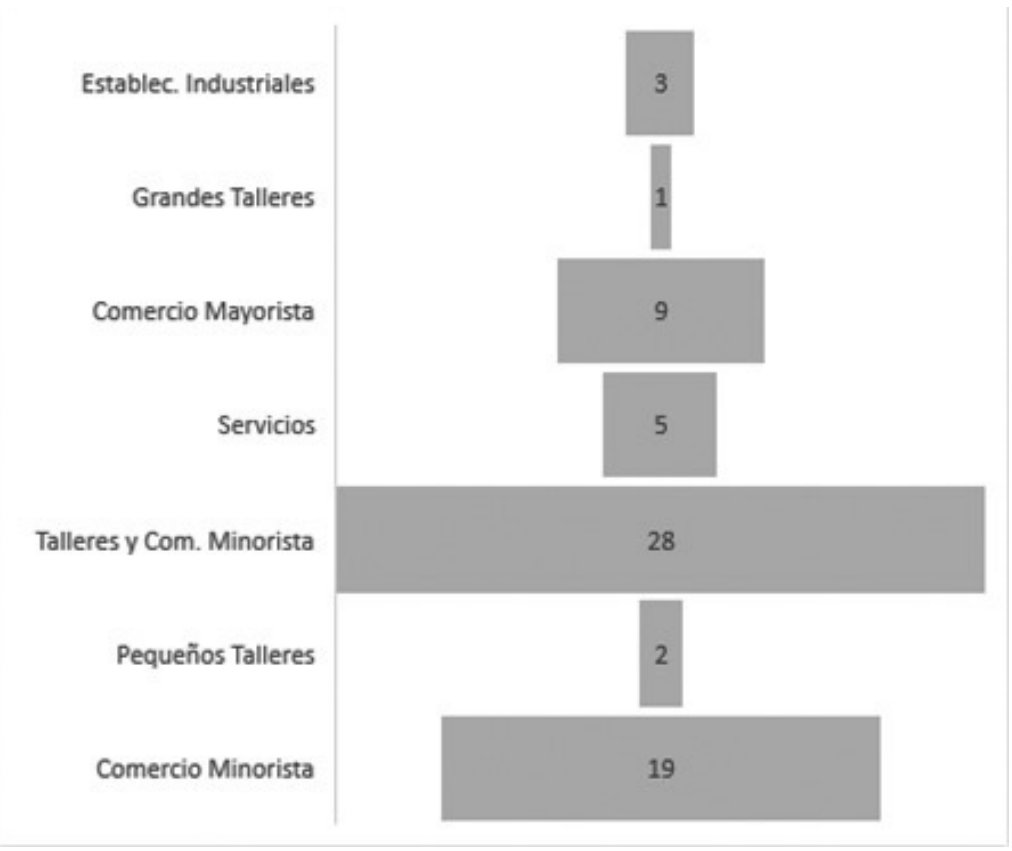


En una segunda etapa, que ya está representada en los dos grupos inferiores, y gracias a los ahorros logrados en el periodo previo, algunos de estos comerciantes ambulantes se asentaban en el Plan de Valparaíso o en Viña del Mar, alquilando un local modesto, que en su trastienda o en su segundo piso muchas veces también servía de residencia familiar. Aquí, la venta seguía siendo minorista, pero ahora se incorporaba el objetivo de posicionar la tienda y conseguir una clientela estable que concurriera habitualmente al local. En otros casos, en esta etapa el inmigrante de primera generación asumía el papel de pequeño fabricante a través de un diminuto taller o instalaba una modesta residencial. Por cierto, contraían vínculos entre ellos, como con otros que eran fabricantes mayores, comerciantes mayoristas o importadores.

En tercer término, si tenían éxito, y dependiendo de las oportunidades relativas que ofrecieran ciertos tipos de eslabonamientos, podían plantearse encadenar hacia atrás y sumar a su actividad comercial la fabricación a pequeña escala. De ahí, la importante presencia del pequeño taller que, a la vez, era una tienda al menudeo, donde probablemente se vendían artículos propios y otros comprados a mayoristas. Al mismo tiempo, quien fuera profesional independiente con estudios universitarios podía desarrollar de manera complementaria alguna actividad comercial o como pequeño fabricante, ocupando el mismo domicilio profesional o instalando un pequeño establecimiento comercial o manufacturero en la cercanía.

En una cuarta fase, y siempre suponiendo éxito en la etapa anterior, podía: 1) Plantearse establecer una sucursal de comercio minorista en Valparaíso o en ciudades cercanas (lo que volvía a elevar el número de comercios minoristas en la base de la pirámide, o subir hacia sus grupos superiores; 2) Adquiriendo un inmueble de mayor tamaño en Valparaíso o Viña del Mar, donde en función de las mayores ventajas relativas, podía ensanchar su actividad comercial o de servicios (ya que también podía tratarse de una hospedería o pensión), levantando un gran taller manufacturero, crear derechamente una industria, o desarrollando el comercio mayorista, de distribución o de importaciones, muchas veces manteniendo un encadenamiento hacia atrás mediante la venta al detalle.

Por otro lado, el gráfico 3 también revela que hacia 1944 había un número importante de tiendas de comercio minorista (19) y unos escasos pequeños talleres (2), que se hallan en la segunda etapa -quizá asociados a más personas de reciente inmigración- mientras que había un núcleo mayoritario de pequeños talleres-tienda (28) y unos pocos profesionales independientes (3), que se sitúan en una tercera fase acumulativa de experiencia y de logros; finalmente, existía un grupo no despreciable de grandes comerciantes (9), y un número muy reducido de grandes talleres manufactureros (1) y establecimientos industriales propiamente tal (3). Esto significa que hacia 1944 la mayor parte de estos sesenta y cinco empresarios (43) había alcanzado un grado mínimo de consolidación, si se asigna al pequeño taller-tienda el piso mínimo para esa categoría, pero que, al mismo tiempo, los desplazamientos obedecían a una cierta demografía empresarial muy exigente y selectiva.

En cuanto a su localización, el gráfico 4 muestra que un elevado porcentaje de los establecimientos (62,7\%) se localizaba en cuatro calles del Plan de Valparaíso (Victoria, Condell, Pedro Montt y Esmeralda). 
GRÁFICO 4

Calles más frecuentes (por $N^{\circ}$ de establecimientos)

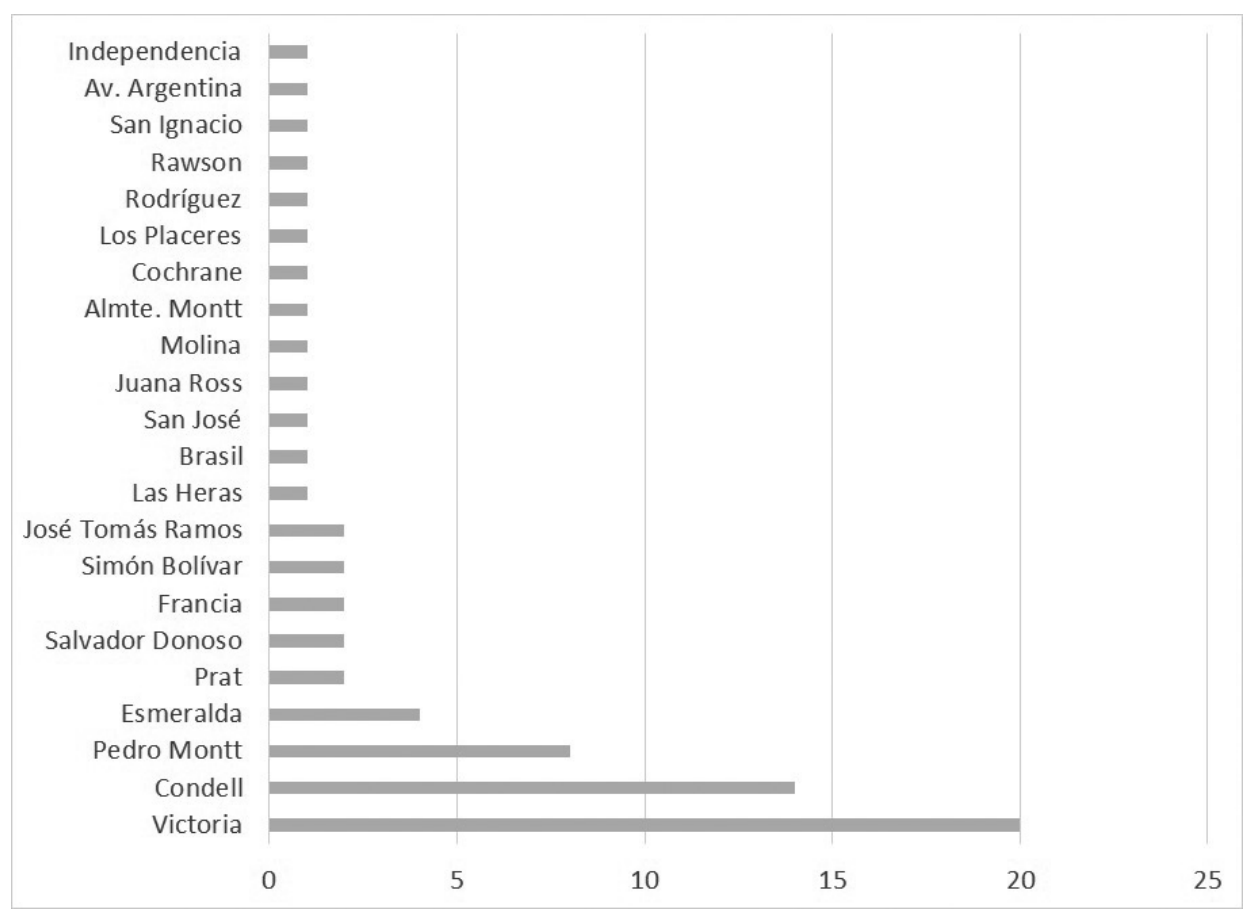

Pero, ¿qué particularidad tenían estas calles? Para apreciarlo, será necesario observar a través de la imagen 1 la disposición de estas calles en el Plan de Valparaíso, usando la actual numeración y configuración de las calles -que no han cambiado esencialmente. Aunque tres establecimientos localizados en Viña del Mar tuvieron un impacto muy marginal, fueron listados al final para fines ilustrativos. Por otro lado, la imagen 1 incluye seis establecimientos en Valparaíso que aportaban dirección, pero no contaban con otros datos, así como un establecimiento perteneciente a dos hermanos de segunda generación (Grossman).

De aquí se puede deducir que hacia 1944 los inmigrantes judíos se concentraban esencialmente en Valparaíso, a tono con la prevalencia que esa ciudad tenía sobre Viña del Mar en esos años. Así y todo, los tres establecimientos localizados en Viña del Mar (dos hospederías y una sucursal de peletería) revelan los inicios de un proceso que con los años culminaría con el mayor dinamismo de Viña del Mar y el sucesivo traslado a ella de buena parte de la comunidad judía de Valparaíso.

Además, se revela que proyectadas hacia el sur a través de las calles Condell, Esmeralda y Salvador Donoso, las calles Victoria, Condell, Pedro Montt y Esmeralda servían y siguen operando como vías longitudinales de Valparaíso, por las que se trasladan miles de personas todos los días, deteniéndose en ciertos puntos para adquirir algún producto o realizar algún trámite, para luego reanudar el viaje. Se trata de arterias que ya en 
esos años movilizaban a una importante población flotante, muy heterogénea social y laboralmente, que constituía en sí misma un mercado muy potente, que demandaba desde artículos muy modestos y baratos hasta bienes de lujo y servicios de alta cualificación. Por otro lado, Valparaíso todavía conservaba dinamismo productivo y comercial, por lo que no habría sido sensato asentar los establecimientos en Viña del Mar, que en esos años recién despuntaba como ciudad dormitorio.

De cualquier modo, esta disposición de los establecimientos revela que se trataba de emprendimientos muy variados, que no podían acotar sus segmentos de consumidores al alto grupo social, sino, más bien, debían ser capaces de llegar a la demanda de los grupos mesocráticos e, incluso, populares. Por eso, no podían localizarse en un barrio o sector acotado de altos ingresos, como podía serlo cerro Alegre, por ejemplo, y ni siquiera a un rectángulo definido, delimitado por ciertas manzanas.

\section{CARACTERÍSTICAS ESENCIALES DE SESENTA Y CINCO EMPRESARIOS JUDÍOS DEL PERIOdo 1920-1944 EN Valparaíso y Viña del Mar}

En este apartado, de estos sesenta y cinco empresarios judíos se intentó determinar su origen étnico, quienes tenían estudios universitarios y el tiempo que demoraron en instalar un segundo establecimiento en Valparaíso-Viña del Mar o en otras ciudades.

De acuerdo con los gráficos 5 y 6, la mayor parte de los empresarios de origen judío entre 1920 y 1944 en Valparaíso y Viña del Mar era de origen askenazí (80\%), correspondiendo un $16,9 \%$ al grupo sefardí y siendo desconocido el restante 3,1\%. En cualquier caso, la participación sefardí en negocios fue proporcionalmente mayor en esta época. Si se toma una muestra general más amplia de 1.833 personas de origen judío para Valparaíso y Viña del Mar entre 1905 y 2015, esta arroja apenas un 4,6\% sefardí frente a un 95,2\% askenazí. Probablemente este menor protagonismo sefardí en un periodo más extendido se deba a que luego de las Leyes de Nüremberg de 1935, y su posterior endurecimiento, el flujo de refugiados judíos de origen alemán (azkenazíes) fue predominante. En efecto, solo entre 1939 y 1940 arribaron alrededor de trece mil personas provenientes del Tercer Reich ${ }^{12}$.

Sin duda, la abrupta llegada de alrededor de doce mil refugiados en $1939^{13}$ alteró completamente las participaciones porcentuales entre askenazíes y sefardíes, no solo en Valparaíso y Viña del Mar sino a escala nacional. De hecho, el registro de empresarios en 1944 debió estar relativamente afectado por ese flujo, ya que incluyó a empresarios que ya habían logrado fundar al menos un establecimiento, algo que pudieron lograr algunos de los nuevos refugiados. Esto resalta el protagonismo que los sefardíes lograron mantener en los negocios - dentro del universo judío- en Valparaíso y Viña del Mar hasta 1944, pero también adelanta que no tenían posibilidades de sostenerlo más adelante.

\footnotetext{
${ }^{12}$ Matus, op. cit., p. 67.

${ }^{13}$ Irmtrud Wojak, "Chile y la inmigración judeo-alemana”, en Avraham Milgram (ed.), Entre la aceptación y el rechazo. América Latina y los refugiados del nazismo, Jerusalén, Yad Vashem, 2003, p. 162. Véase también, de la misma autora, Exile in Chile. Die deutsch-jüdische und politische emigration während des Nationalsozialismus, 1933-1945, Berlin, Metropol Verlag, 1994, p. 57.
} 


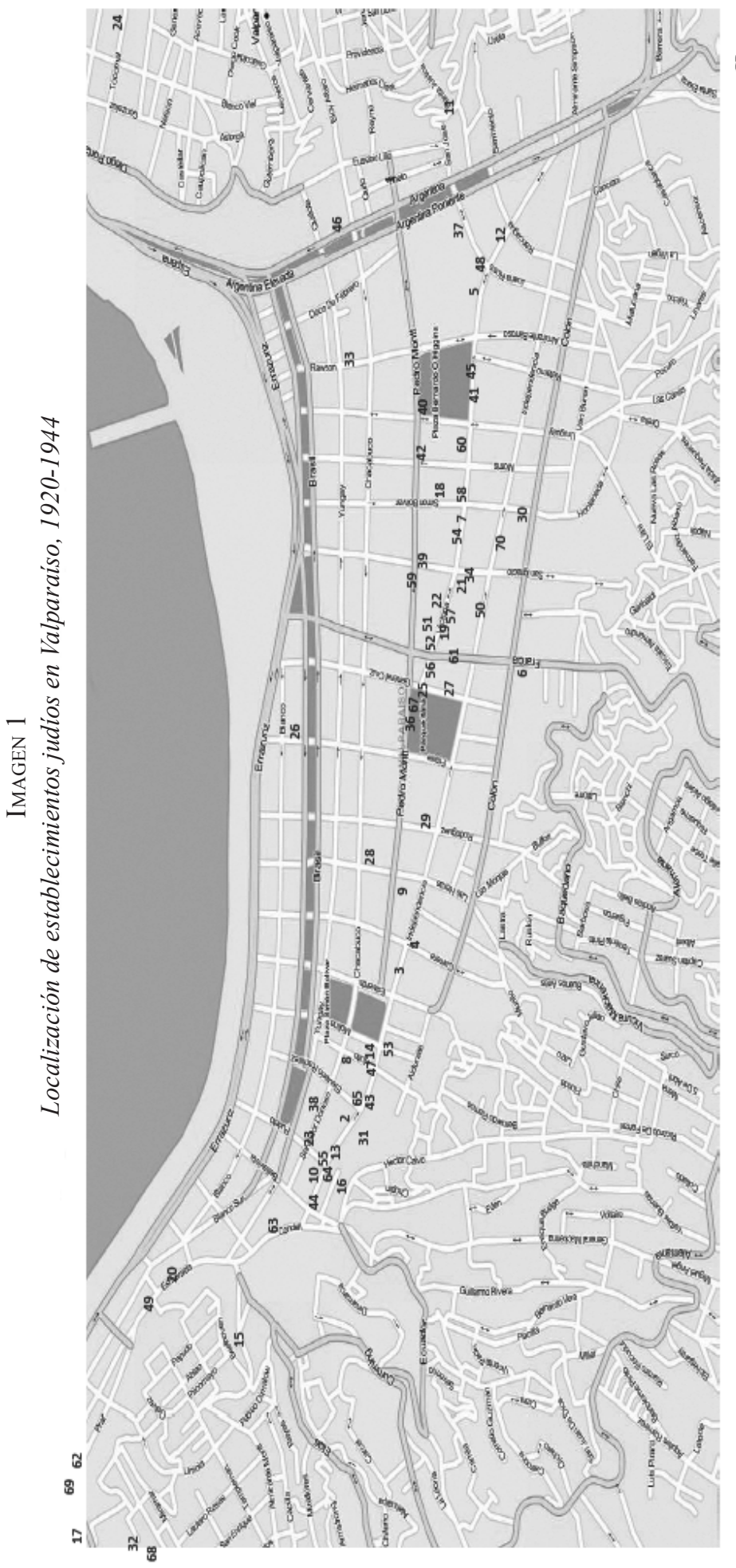

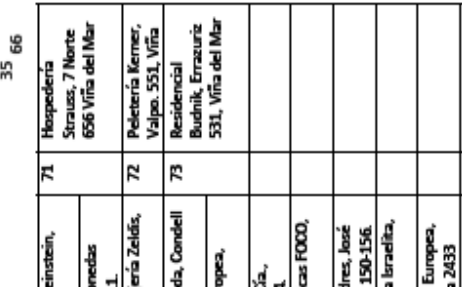

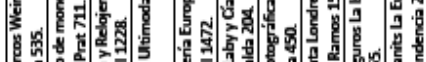
10

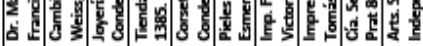

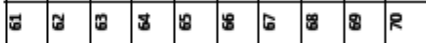

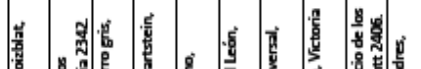

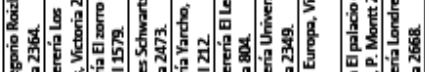

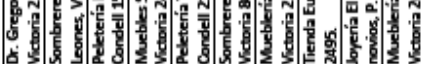
$\approx$ \#

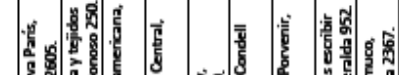

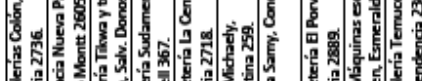

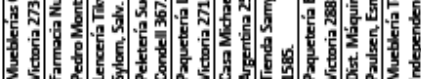

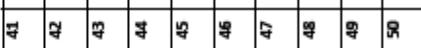

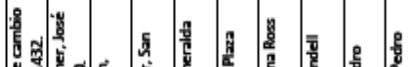

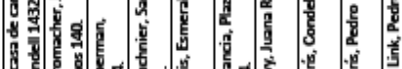

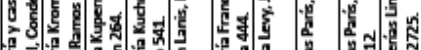

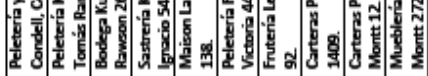

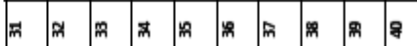

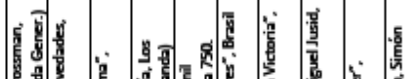

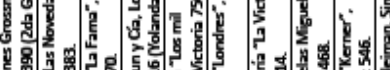

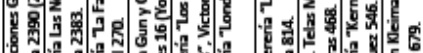

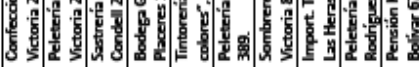

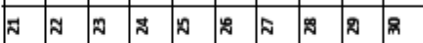

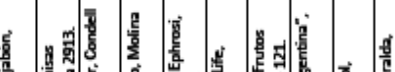

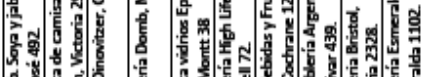

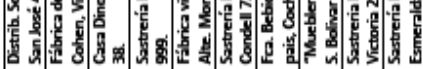
$\Rightarrow \Rightarrow \Rightarrow D=A \Rightarrow 2 R$

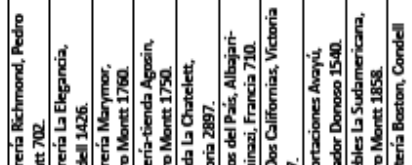

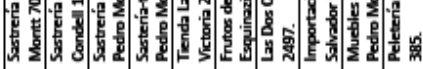

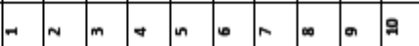




\section{GRÁFICO 5}

Pertenencia étnica en negocios, Valparaíso-Viña 1944 (\%)

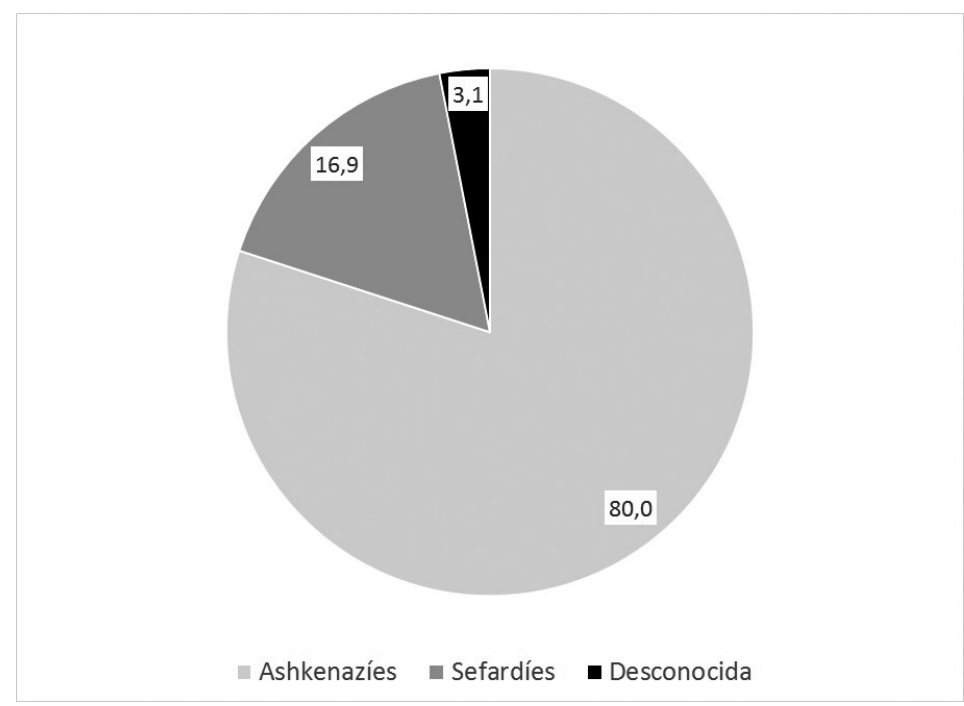

GrÁFICO 6

Pertenencia étnica en general, Valparaíso-Viña 1944 (\%)

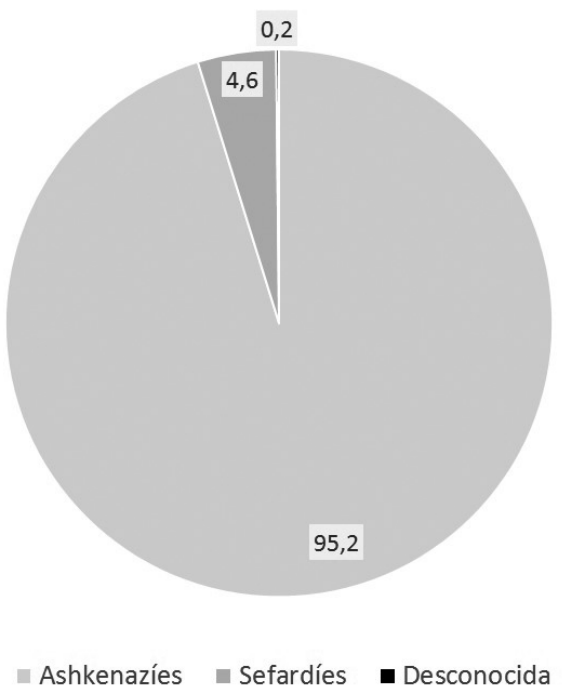

En cuanto a grado de educación terciaria, solo consta de cuatro personas con estudios universitarios completos o incompletos (Juan Guiloff, que había llegado en 1920 y cursaba Ortodoncia en $1^{\text {er }}$ año; Clara Litvak de Hirsch, que también llegó con once años en 1920 y que se había titulado como química farmacéutica; Gregorio Roizblatt, que habiendo llegado 
hacia 1925 ejercía como psiquiatra y, finalmente, Marcos Weinstein, que llegó al país en 1915 y se tituló como médico en 1928). Este bajo porcentaje de empresarios con estudios universitarios $^{14}(6,1 \%)$ es característico de una primera generación de inmigrantes a inicios de siglo Xx, en que, salvo situaciones muy excepcionales, el acceso a los estudios universitarios era aún bastante reducido. Lo interesante es que, a excepción de Juan Guiloff-que al parecer llegó adulto y con sus estudios realizados- los otros tres casos llegaron a una edad menor e hicieron sus estudios universitarios en el país, lo que revela una rápida inserción local.

En cualquier caso, esta cifra también ayuda a caracterizar a esta oleada de inmigración judía a Valparaíso y Viña del Mar como personas que en su gran mayoría tenían educación primaria completa $\mathrm{y}$, en alguna medida, secundaria completa, cursadas en sus países de origen.

En relación con el tiempo que tardaron estos inmigrantes en conseguir una relativa prosperidad, se consiguió información relativa a setenta y un establecimientos, de los cuales sesenta y dos de ellos fueron un primer emprendimiento y los nueve restantes conformaron un segundo establecimiento o sucursal del primero, ya fuera en ValparaísoViña del Mar, o en otras ciudades ${ }^{15}$. La creación de estos establecimientos aparece consignada en el gráfico 7 , donde se recogieron estos setenta y uno de acuerdo con la primera fecha en que aparecen registrados en alguna fuente primaria.

GrÁFICO 7

Registro de establecimientos, 1919-1944

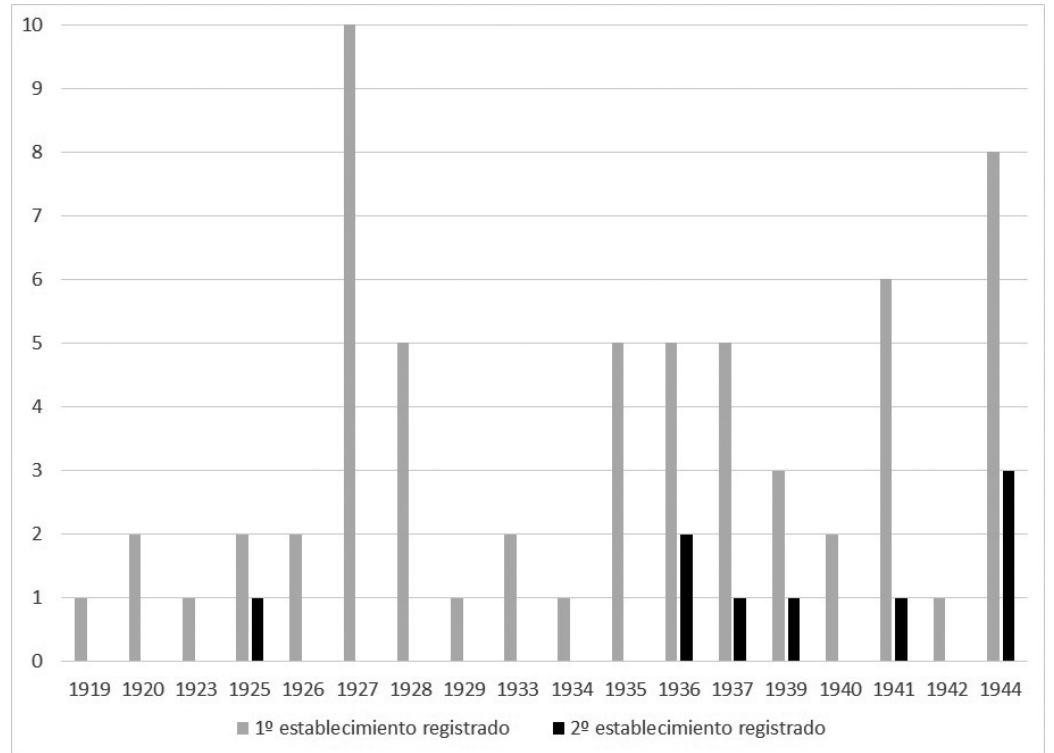

\footnotetext{
${ }^{14}$ Que podría estar distorsionado a la baja, por ausencia de datos para los otros sesenta y un casos.

${ }^{15}$ El número de establecimientos es levemente superior a los sesenta y siete registrados en Valparaíso y Viña del Mar porque incluye otros establecimientos y sucursales localizadas en otras ciudades cercanas (Santiago y Quillota, esencialmente). Esta misma razón extiende el periodo de estudio retrospectivamente hasta 1919, para incluir un establecimiento situado en Santiago ese año.
} 
Una primera lectura del gráfico 7 coincide bastante con la trayectoria general de estos emprendedores, que antes se sintetizó en el apartado dedicado a la escala de las operaciones. La escasez de establecimientos registrados entre 1919 y 1926 puede deberse a que en esa primera etapa estos emprendedores ejercían un comercio minorista ambulante, con ventas a plazo, porque aún no estaban en condiciones de alquilar o comprar un inmueble para estos fines. En ese sentido, el hecho que Isaac Smirnoff contara con dos establecimientos en 1925 fue excepcional (cuadro 3). Pero ya entre 1927 y 1928 se puede observar un importante número de nuevos establecimientos, que indica que ya en esa segunda etapa algunos de estos emprendedores habían adquirido un grado significativo de prosperidad, al punto que muchos de esos establecimientos eran de su plena propiedad. Por otro lado, se debe recordar que la gran mayoría de estos inmuebles deben haber sido de modesto tamaño, ya que en su mayor parte eran pequeñas tiendas, talleres o tiendas-taller, que en muchos casos servían también de residencia. El bajo número de establecimientos fundados entre 1929 y 1934, sin duda, debe estar asociado al impacto de la Gran Depresión en Chile, pero también puede haber sido un paréntesis razonable antes de que comenzara una nueva etapa entre 1935 y 1944, lapso en que no solo se reanudó con firmeza la creación dde nuevas actividades productivas sino que, además, se comienza a advertir la prosperidad de un modo más notorio, en la medida en que aquí se concentra la fundación de un segundo establecimiento o sucursal, ya fuera en ValparaísoViña del Mar, como en otras ciudades cercanas (Santiago y Quillota). Por otra parte, el reimpulso a la instalación de un primer establecimiento, especialmente entre 1939 y probablemente 1944 también recoge la llegada de refugiados provenientes del Tercer Reich.

En esa última fase, en paralelo a la aparición de sucursales y a un segundo establecimiento, el comercio minorista y la fabricación en pequeña escala había dado paso a inmuebles de mayor tamaño dedicados a actividad comercial mayorista, a importaciones, distribución, servicios a una mayor escala o a fábricas.

\section{CuAdro 3}

Fundación de establecimientos por año

(incluye sucursales fuera de Valparaíso-Viña)

\begin{tabular}{|c|c|c|c|}
\hline Año & & Establecimiento & Dueño(s) \\
\hline 1919 & 1 & Peletería de José Medvinsky (Stgo) & José Medvinsky (1) \\
\hline \multirow[t]{2}{*}{1920} & \multirow[t]{2}{*}{2} & Peletería Londres & Elías Horovitz \\
\hline & & Sombrerería La Victoria & Cecilia Jajam \\
\hline 1923 & 1 & Tienda Las dos californias & Alberto e Isaac Avayú (1) \\
\hline \multirow[t]{3}{*}{1925} & \multirow{3}{*}{3} & Fábrica de bebidas gaseosas & Isidoro Froimovich (1) \\
\hline & & Gran peletería Yarcho & Isaac Smirnoff (1) \\
\hline & & Peletería-sombrerería El León & Isaac Smirnoff (2) \\
\hline \multirow[t]{2}{*}{1926} & \multirow[t]{2}{*}{2} & Lenc.Tikwa/ imp. Sylom (1926) & Jaime Mandel (1) \\
\hline & & Sucursal en Santiago & Jaime Mandel (2) \\
\hline \multirow{4}{*}{1927} & \multirow{4}{*}{4} & Sastrería femenina Marymor & Moisés Abramson \\
\hline & & Peletería Boston & Samuel Bronfman \\
\hline & & Sastrería High Life & José Finkelstein \\
\hline & & Sastrería La Fama & Juan Guiloff \\
\hline
\end{tabular}




\begin{tabular}{|c|c|c|c|}
\hline \multirow{6}{*}{1927} & \multirow{6}{*}{6} & Tintorería Los mil colores & M. Heller \\
\hline & & Importadora de telas Miguel Jusid & Miguel Jusid \\
\hline & & Peletería Francia & Gregorio Levinson \\
\hline & & Fca. de carteras Paris, Condell & Victor Lifschitz (1) \\
\hline & & Peletería El zorro gris & Felipe Schapira \\
\hline & & Mueblería y tapicería Londres & José y Julio Weinstein \\
\hline \multirow[t]{5}{*}{1928} & \multirow{5}{*}{5} & Sastrería Richmond & L. Abramovich \\
\hline & & Sastrería La Elegancia & Moisés Abramson \\
\hline & & Casa Dinovitzer & Bernardo Dinovitzer \\
\hline & & Peletería Condell & Bernardo Kramarenko \\
\hline & & Maison Lanis venta de arts. de lujo & David Lanis \\
\hline 1929 & 1 & Sastrería Bristol & Samuel Grimblatt \\
\hline \multirow[t]{2}{*}{1933} & \multirow[t]{2}{*}{2} & Sastrería Agosin & Marcos Agosin \\
\hline & & Frutos del país & Salvador Albajari y Moreno Esquenazi \\
\hline 1934 & 1 & Residencial Budnik (Viña del Mar) & Budnik \\
\hline \multirow[t]{5}{*}{1935} & \multirow{5}{*}{5} & Sastrería Kuschnier & Carlos Kuschnier \\
\hline & & Frutería Levy & Jaime Levy \\
\hline & & Paquetería La Central & Alberto Messina \\
\hline & & Paquetería El Porvenir & Marcos Nahum \\
\hline & & Dr. Consulta médica & Gregorio Roizblatt (1) \\
\hline \multirow[t]{7}{*}{1936} & \multirow{7}{*}{7} & Peletería y tintorería Las Novedades & Benjamín Grossman \\
\hline & & Peletería y tienda Kerner & Aron y Máximo Kerner \\
\hline & & Sucursal tienda Kerner, Viña del Mar & Aron y Máximo Kerner \\
\hline & & Pensión Kleiman & Marcos Kleiman \\
\hline & & Cambio de monedas & Bernardo Kramarenko (2) \\
\hline & & Sucursal Fábrica de Carteras Stgo. & Victor Lifschitz (2) \\
\hline & & Casa Weiss, casa de cambio de monedas & Weiss \\
\hline \multirow[t]{6}{*}{1937} & \multirow{6}{*}{6} & Impor. y confección arts. de lana y algodón & Alberto e Isaac Avayú (2) \\
\hline & & Fca. de muebles La Sudamericana & Jacobo Blinder \\
\hline & & Distribución de soya y jabón & Bernardo Burstein \\
\hline & & Fábrica de camisas Cohen & Salomón Cohen \\
\hline & & Domb y Cía. & Domb \\
\hline & & Paulsen y Cía. Distrib. Máquinas escribir & Paulsen \\
\hline \multirow[t]{4}{*}{1939} & \multirow{4}{*}{4} & Bodega frutos del país & Isidoro Froimovich (2) \\
\hline & & Fábrica de muebles La Argentina & Adolfo Gomberoff \\
\hline & & Bodega Kuperman & Isaac Kuperman \\
\hline & & Tienda Europa & Alberto e Isaac Ventura \\
\hline \multirow[t]{2}{*}{1940} & \multirow[t]{2}{*}{2} & Fca.vidrios-cristales. Ind. vidrios Ltda. & Alfredo Ephrosi y Ricardo Freudenberg \\
\hline & & Artefactos sanitarios La Europea & Emmanuel Hirnheimer \\
\hline \multirow[t]{7}{*}{1941} & \multirow{7}{*}{7} & Mueblerías Colón & León Link (1) \\
\hline & & Farmacia Nueva París & Clara Litvak de Hirsch \\
\hline & & Casa-multitienda Michaely & Samuel Michaely (1) \\
\hline & & Mueblería Temuco & José Rocinsky \\
\hline & & Peletería y sombrerería Los Leones & Gregorio Roizblatt (2) \\
\hline & & Joyería-relojería El palacio de los novios & Mauricio Volosky \\
\hline & & Dr. Consulta médica & Marcos Weinstein \\
\hline 1942 & 1 & Productos de cuero Kromacher & Sigfredo y Ernesto Kromacher \\
\hline
\end{tabular}




\begin{tabular}{|c|c|c|c|}
\hline \multirow[t]{11}{*}{1944} & \multirow{11}{*}{11} & Sastrería Agosin, suc. Quillota (1944) & Marcos Agosin \\
\hline & & Sastrería Esmeralda & Samuel Grimblatt \\
\hline & & Tienda de lujo La Chatelett & Victor Alaluf \\
\hline & & Gun y Cía. Frutos del país y abarrotes & Gun \\
\hline & & Mueblería Link & León Link (2) \\
\hline & & Sucursal Stgo. de Importadora Sylom & Jaime Mandel (2) \\
\hline & & Tienda Samy & Samuel Michaely (2) \\
\hline & & Muebles Shwartstein & Mauricio Schwartstein \\
\hline & & Hospedería Strauss & Max y Alejandro Strauss \\
\hline & & Mueblería Universal & Alberto Sverdlov \\
\hline & & Joyería-relojería Election, palacio de relojes & Salomón Zeldis \\
\hline Total & 71 & & \\
\hline
\end{tabular}

Esta trayectoria ostenta diferencias importantes con la hipótesis inicial asociada. En efecto, ella demuestra que la prosperidad como fenómeno mensurable a través de la fundación de un segundo establecimiento o por medio de la transición a una mayor escala de operaciones no se consiguió rápidamente -entendiendo como tal un rango de cincosiete años-, ya que al menos transcurrieron diez años entre 1927-1928 y 1936-1944 para que se obtuvieran tales logros.

Esta constatación también sirve para enfatizar que esta primera década de permanencia en el país debe haber significado gran esfuerzo y sacrificio para la mayor parte de los sesenta y cinco inmigrantes estudiados y resalta otro rasgo típico de todo emigrante: el que emigra por lo general está dispuesto a hacer un esfuerzo mucho mayor al que habría realizado en su país de origen, básicamente porque su horizonte de expectativas es bastante superior.

GRADO DE COMPROMISO Y APOYO AL FORTALECIMIENTO COMUNITARIO MOSTRADO POR ESTOS SESENTA Y CINCO EMPRESARIOS JUDÍOS DURANTE EL PERÍODO 1920-1944 en Valparaíso y Viña del Mar

¿En qué medida el paulatino progreso material de estos inmigrantes judíos arribados a Valparaíso y Viña del Mar entre 1920 y 1944 pudo haber colaborado a sentar las bases de la red de organizaciones comunitarias judías en tales ciudades? El cuadro 4 entrega la información basal y los gráficos $8,9,10$ y 11 la procesan.

CuAdro 4

Grado de asociatividad y compromiso comunitario

\begin{tabular}{|c|c|c|c|c|c|c|c|}
\hline & & \multicolumn{2}{|c|}{ Donante } & \multicolumn{3}{|c|}{ Participación en organizaciones } & \multirow[b]{2}{*}{ Sepultado en } \\
\hline & & $\begin{array}{c}\text { Edificio } \\
\text { SUIV }\end{array}$ & $\begin{array}{c}\text { Keren } \\
\text { Hayesod }\end{array}$ & 1 & 2 & 3 & \\
\hline 1 & L. Abramovich & & & & & & \\
\hline 2 & Moisés Abramson & $\mathrm{X}$ & $\mathrm{X}$ & Max Nordau & $\begin{array}{l}\text { Vicepdte } \\
\text { SUIV }\end{array}$ & Socio CIS & P. Ancha \\
\hline 3 & Marcos Agosin & & & $\begin{array}{l}\text { Cargo Max } \\
\text { Nordau }\end{array}$ & $\begin{array}{l}\text { Vicepdte } \\
\text { SUIV }\end{array}$ & & P. Ancha \\
\hline
\end{tabular}




\begin{tabular}{|c|c|c|c|c|c|c|}
\hline 4 & Víctor Alaluf & & & & & P. Ancha \\
\hline 5 & Salvador Albajari & & & Vice SUIV & & P. Ancha \\
\hline 6 & Moreno Esquinazi & & & $\begin{array}{l}\text { Direct. Max } \\
\text { Nordau }\end{array}$ & & P. Ancha \\
\hline 7 & Alberto Avayú & & $\mathrm{X}$ & $\begin{array}{l}\text { Tesor-pte } \\
\text { Max N }\end{array}$ & $\begin{array}{l}\text { Keren } \\
\text { Kayemet }\end{array}$ & \\
\hline 8 & Isaac Avayu & & & & & \\
\hline 9 & Jacobo Blinder & $\mathrm{X}$ & $\mathrm{X}$ & $\begin{array}{l}\text { Fund y pte } \\
\text { SUIV }\end{array}$ & & El Belloto \\
\hline 10 & Samuel Bronfman & & & & & \\
\hline 11 & Budnik & & & & & \\
\hline 12 & Bernardo Burstein & $\mathrm{X}$ & $\mathrm{X}$ & $\begin{array}{l}\text { Tesor.y rev } \\
\text { SUIV }\end{array}$ & Soc. CIS & \\
\hline 13 & Salomón Cohen & & & $\begin{array}{l}\text { Cargo Max } \\
\text { Nordau }\end{array}$ & & P. Ancha \\
\hline 14 & Bernardo Dinovitzer & $\mathrm{X}$ & & $\begin{array}{l}\text { Vice y sec } \\
\text { SUIV }\end{array}$ & & P. Ancha \\
\hline 15 & Domb & & & & & \\
\hline 16 & Alfredo Ephrosi & & & & & \\
\hline 17 & Ricardo Freudenberg & & & & & El Belloto \\
\hline 18 & José Finkelstein & & & $\begin{array}{l}\text { Soc. Max } \\
\text { Nordau }\end{array}$ & & El Belloto \\
\hline 19 & Isidoro Froimovich & $\mathrm{X}$ & $\mathrm{X}$ & $\begin{array}{l}\text { Voc. y rev } \\
\text { SUIV }\end{array}$ & & P. Ancha \\
\hline 20 & Adolfo Gomberoff & & & $\begin{array}{l}\text { Soc. Max } \\
\text { Nordau }\end{array}$ & & El Belloto \\
\hline 21 & Samuel Grimblatt & $\mathrm{X}$ & $\mathrm{X}$ & $\begin{array}{l}\text { Tesor.y vocal } \\
\text { SUIV }\end{array}$ & & El Belloto \\
\hline 22 & Benjamín Grossman & $\mathrm{X}$ & & Soc. SUIV & & El Belloto \\
\hline 23 & Juan Guiloff & $\mathrm{X}$ & $\mathrm{X}$ & $\begin{array}{l}\text { Voc, Sec y } \\
\text { rev.SUIV }\end{array}$ & $\begin{array}{l}\text { Club Dep- } \\
\text { Cult Isr. }\end{array}$ & \\
\hline 24 & Gun & & & & & \\
\hline 25 & M. Heller & & & & & \\
\hline 26 & Emmanuel Hirnheimer & & & & & \\
\hline 27 & Elías Horovitz & & & & & \\
\hline 28 & Cecilio Jajam & $\mathrm{X}$ & $\mathrm{X}$ & Voc. SUIV & & \\
\hline 29 & Miguel Jusid & $\mathrm{X}$ & $\mathrm{X}$ & $\begin{array}{l}\text { Soc. Max } \\
\text { Nordau }\end{array}$ & SUIV & \\
\hline 30 & Aron Kerner & $\mathrm{X}$ & & Soc. SIV & & El Belloto \\
\hline 31 & Máximo Kerner & $\mathrm{X}$ & & Soc. SUIV & & \\
\hline 32 & Marcos Kleiman & & & Soc. SUIV & & P. Ancha \\
\hline 33 & Bernardo Kramarenko & $\mathrm{X}$ & $\mathrm{X}$ & $\begin{array}{l}\text { Tesor.y vice } \\
\text { SUIV }\end{array}$ & & \\
\hline 34 & Sigfredo Kromacher & & & & & \\
\hline 35 & Ernesto Kromacher & & & & & \\
\hline 36 & Isaac Kuperman & $\mathrm{X}$ & & Soc. SUIV & & El Belloto \\
\hline 37 & Carlos Kushnier & $\mathrm{X}$ & & Voc. SUIV & & \\
\hline 38 & David Lanis & $\mathrm{X}$ & $\mathrm{X}$ & Voc. SUIV & $\begin{array}{l}\text { Deleg. Cong } \\
\text { Sion }\end{array}$ & P. Ancha \\
\hline
\end{tabular}




\begin{tabular}{|c|c|c|c|c|c|c|}
\hline 39 & Gregorio Levinson & $\mathrm{X}$ & $\mathrm{X}$ & Fund. SUIV & $\begin{array}{l}\text { Sec. Club } \\
\text { Macabeos }\end{array}$ & \\
\hline 40 & Jaime Levy & & & $\begin{array}{l}\text { Sec Max } \\
\text { Nordau }\end{array}$ & & P. Ancha \\
\hline 41 & Víctor Lifschitz & $\mathrm{X}$ & $\mathrm{X}$ & $\begin{array}{l}\text { Pte y rev } \\
\text { SUIV }\end{array}$ & $\begin{array}{l}\text { Pdte Hon } \\
\text { CIS }\end{array}$ & \\
\hline 42 & León Link & & & Vice SUIV & & \\
\hline 43 & Clara Litvak de Hirsch & & & & & El Belloto \\
\hline 44 & Jaime Mandel & & & \begin{tabular}{|l} 
Vice \\
Juventud \\
Sion. \\
\end{tabular} & & \\
\hline 45 & José Medvinsky & & & & & \\
\hline 46 & Alberto Messina & & & $\begin{array}{l}\text { Soc. Max } \\
\text { Nordau }\end{array}$ & & P. Ancha \\
\hline 47 & Samuel Michaely & & $\mathrm{x}$ & Vice SUIV & $\begin{array}{l}\text { Del. Cong } \\
\text { Sion }\end{array}$ & El Belloto \\
\hline 48 & Marcos Nahum & & & $\begin{array}{l}\text { Protesor- } \\
\text { voc. MaxN }\end{array}$ & & \\
\hline 49 & Paulsen & & & & & \\
\hline 50 & José Rocinsky & & $X$ & Tesor SUIV & & \\
\hline 51 & Gregorio Roizblatt & & $\mathrm{X}$ & Tesor SUIV & & P. Ancha \\
\hline 52 & Felipe Schapira & $\mathrm{x}$ & $\mathrm{X}$ & $\begin{array}{l}\text { Fun-voc-vic- } \\
\text { Pte SUIV }\end{array}$ & $\begin{array}{l}\text { Del. Cong. } \\
\text { Sion }\end{array}$ & \\
\hline 53 & Mauricio Schwartstein & $X$ & $\mathrm{X}$ & Soc. SUIV & & El Belloto \\
\hline 54 & Isaac Smirnoff & $\mathrm{X}$ & $\mathrm{X}$ & $\begin{array}{l}\text { Pte SUIV- } \\
\text { Com.InmIsr }\end{array}$ & Soc. CIS & El Belloto \\
\hline 55 & Max Strauss & & & & & El Belloto \\
\hline 56 & Alejandro Strauss & & & & & El Belloto \\
\hline 57 & Alberto Sverdlov & $\mathrm{X}$ & $\mathrm{X}$ & Socio SIUV & & \\
\hline 58 & Alberto Ventura & & $\mathrm{x}$ & $\begin{array}{l}\text { Cargo Max } \\
\text { Nordau }\end{array}$ & & \\
\hline 59 & Isaac Ventura & & $\mathrm{X}$ & $\begin{array}{l}\text { Pte Max } \\
\text { Nordau }\end{array}$ & & P. Ancha \\
\hline 60 & Mauricio Volosky & & $\mathrm{X}$ & $\begin{array}{l}\text { Sec y Rev } \\
\text { SIUV }\end{array}$ & & \\
\hline 61 & José Weinstein & $\mathrm{X}$ & $\mathrm{X}$ & Soc. SUIV & & \\
\hline 62 & Julio Weinstein & & $\mathrm{X}$ & $\begin{array}{l}\text { Pte, vice y } \\
\text { rev SUIV }\end{array}$ & & P. Ancha \\
\hline 63 & Marcos Weinstein & & $\mathrm{X}$ & $\begin{array}{l}\text { Voc. SUIV } \\
1928\end{array}$ & & \\
\hline 64 & Weiss & & & & & El Belloto \\
\hline 65 & Salomón Zeldis & & $\mathrm{X}$ & $\begin{array}{l}\text { Cargo N/E } \\
\text { SUIV }\end{array}$ & & El Belloto \\
\hline
\end{tabular}

Siglas y abreviaturas: Max Nordau: Sociedad Israelita Max Nordau (Valparaíso); SUIV: Sociedad Unión Israelita de Valparaíso; Com.InmIsr: Comité Inmigración Israelita; Cong Sion: Congreso Sionista; Juventud Sion.: Juventud Sionista; CIS: Centro Infantil Sionista; Club Dep-Cult Isr: Club Deportivo y Cultural Israelita; Club Los Macabeos: Club Deportivo; Keren Kayemet: Fondo Nacional Judío para la Adquisición de Tierras en Palestina; Keren Hayesod: Fondo Nacional Judío para el Retorno a Israel; Fund: Fundador; Direct.: Director; Pte: Presidente; Pdte Hon: Presidente honorario; Vice: Vicepresidente; Tesor: Tesorero; Protesor: 
Protesorero; Rev: Revisor; Sec: Secretario; Voc: Vocal; Soc: Socio; Voce: Vocero; Del: Delegado; El Belloto: cementerio de El Belloto; P. Ancha: Mausoleo judío del cementerio de Playa Ancha (Valparaíso).

En primer término, las dos columnas del cuadro 4, relativas a donaciones, son una aproximación inicial al grado de interés asociativo y la contribución a esta naciente red de organizaciones comunitarias. En la primera de ellas se recoge quienes aportaron recursos a la construcción del primer edificio de la Sociedad Unión Israelita de Valparaíso $(\mathrm{SUIV})^{16}$, mientras que la segunda registra a quienes aportaron al Keren Hayesod, institución sionista encargada de recaudar fondos para financiar la Aliá (retorno a Israel) de judíos de la Diáspora. Se entiende que la materialización de donaciones revela un tipo de compromiso con la comunidad, ya sea la de la Diáspora, o las de Israel, a través de sus organizaciones respectivas. Los resultados se muestran en el gráfico 8 .

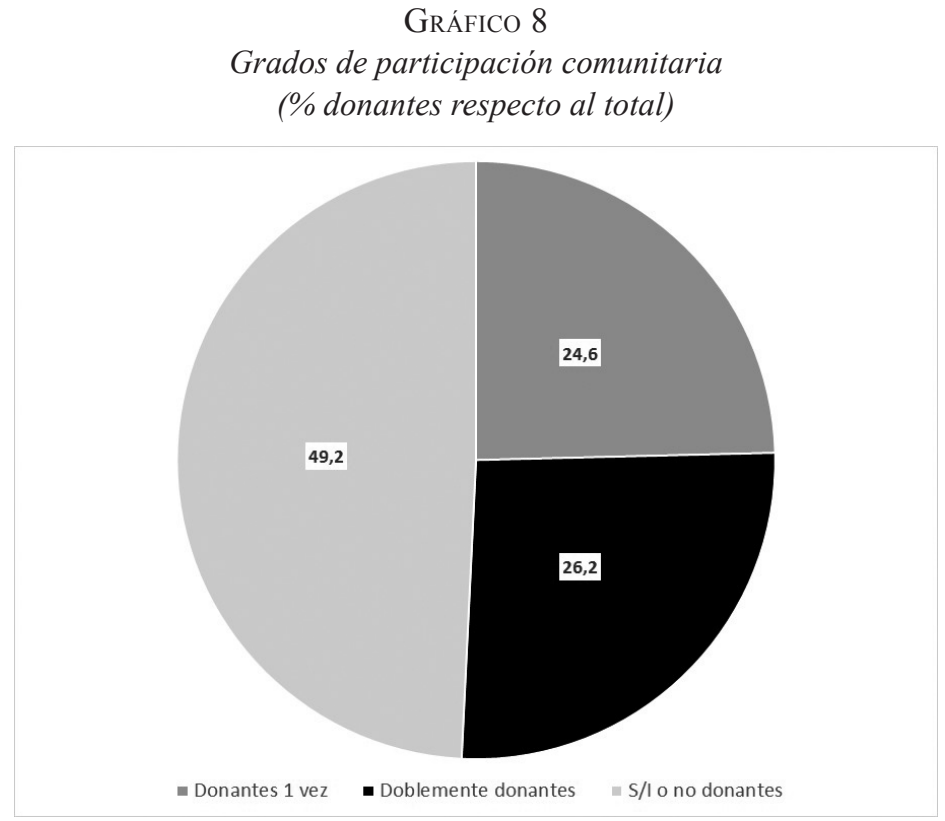

En este caso, puede apreciarse que los que donaron a alguna de ambas causas comunitarias conformaron un 50,8\% del total de las sesenta y cinco personas consideradas, una cifra importante si se considera que recién a fines de la década de 1930 y al cabo de un decenio, llegaron a contar con una posición económica relativamente sólida ${ }^{17}$. Además, llama la atención que un $26,2 \%$ de los considerados actuó como doble donante en estos proyectos.

\footnotetext{
${ }^{16}$ Según Aguirre, op. cit., p. 14, este edificio se habría situado en la Av. Argentina, en la actual localización del Congreso Nacional.

${ }^{17}$ En cualquier caso, el 49,2\% restante puede estar exagerado, debido a la falta de información.
} 
Por su parte, las columnas agrupadas bajo el título "Participación en organizaciones" identifica el tipo de participación de cada uno en un máximo de tres organizaciones. El supuesto es que el desempeño de cargos en las organizaciones comunitarias expresa de otro modo el nivel de compromiso asociativo. Los resultados se recogen en el gráfico 9. Como puede apreciarse, las personas que ocuparon cargos como dirigentes en alguna organización comunitaria llegaron a representar $49,2 \%$ del universo representado, mientras que los que aportan información como dirigentes ocuparon el 50,7\% restante.

\section{GrÁFICO 9 \\ Grados de participación comunitaria (\% dirigentes respecto al total)}

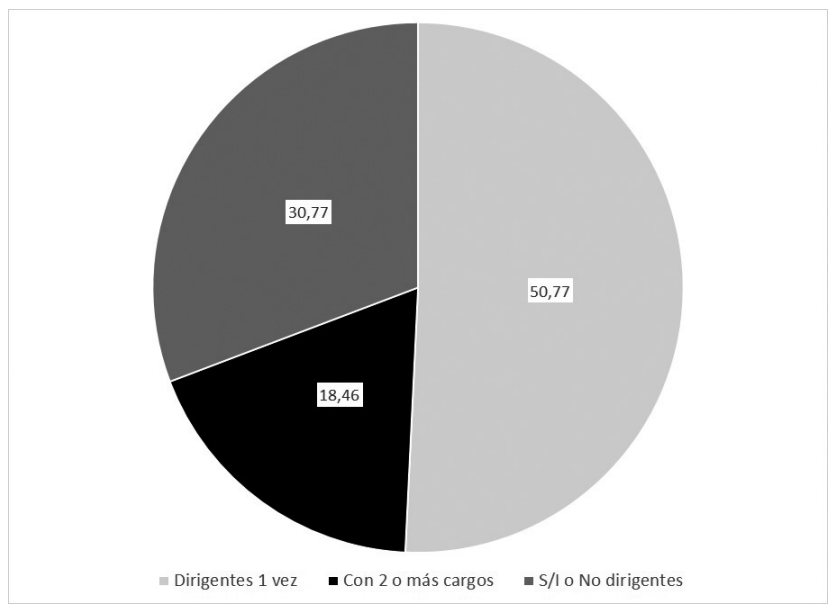

Finalmente, el gráfico 10 recoge los porcentajes de la muestra que fueron sepultados en cementerios comunitarios y aquellos de los que no se dispone de información o fueron sepultados en cementerios no comunitarios. En este caso, se entiende por cementerios comunitarios los mausoleos judíos de los cementerios de Playa Ancha y de El Belloto. Se asume que los que fueron sepultados en mausoleos judíos lo hicieron a través del rito religioso judío, que se cierra con una oración llamada $k a d i s h^{18}$. Esta presencia expresa de otro modo el sentido de pertenencia comunitaria y puede incluirse para evidenciar el nivel de compromiso asociativo.

Una vez más se observa un porcentaje cercano a la mitad de la muestra $(47,7 \%) \mathrm{de}$ personas sepultadas con rito judío, que se contrarresta con un $52,3 \%$ de los que no se tiene información o que cabe la posibilidad que hayan sido sepultados sin rito funerario judío.

\footnotetext{
${ }^{18}$ Que, en cualquier caso, por ser una santificación del nombre de Dios, tiene otros usos y no es una oración meramente acotada a la liturgia funeraria.
} 


\section{GRÁFICO 10 \\ Grados de participación comunitaria (\% sepultados en mausoleos comunitarios respecto al total)}

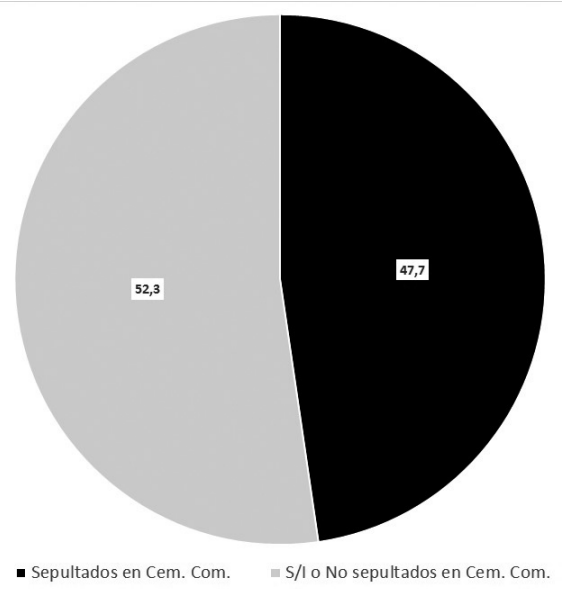

Por otro lado, llama la atención que el porcentaje de donantes sea muy similar (50,8\% del total) al de dirigentes $(49,2 \%)$ o sepultados con ritual judío $(47,7 \%)$, dado que es muy común que cuando una persona está económicamente activa y dispone de escaso tiempo para dedicarlo a actividades asociativas, prefiere donar dinero que cumplir obligaciones como dirigentes, cargos que cuesta llenar ${ }^{19}$. En el caso de las sepultaciones, pueden estar sesgadas a la baja por un importante desplazamiento de familias judías de Valparaíso y Viña del Mar hacia Santiago, que aparece documentada por numerosos testimonios y registros de la vida comunitaria judía en provincias y por lo datos censales $^{20}$. Estas familias pudieron ser sepultadas posteriormente usando un ritual judío, pero en Santiago.

En cualquier caso, un porcentaje de participación cercano al $50 \%$ dentro de una muestra es relativamente elevado, lo que por lo demás no tiene nada de atípico dentro de grupos de inmigrantes directos, que en una primera generación dedican mucho más tiempo y esfuerzos a construir su red asociativa primaria, que funciona de manera normal como una red de apoyo ante todos los desafíos que implica una inserción en un nuevo entorno. Por consiguiente, y como atestigua el gráfico 11, es plausible señalar que el grado de compromiso asociativo de esta primera generación de inmigrantes judíos, residentes en Valparaíso y Viña del Mar entre 1920 y 1944 fue bastante significativa.

\footnotetext{
${ }^{19}$ Laurence R. Iannaccone, "La producción doméstica, el capital humano y la economía de la religión”, en Mariano Tommasi y Kathryn Ierulli (editores), Economía y sociedad, Madrid, Cambridge University Press, 2000, p. 195.

${ }^{20}$ Matus, op. cit., p. 73.
} 


\section{GRÁFICO 11}

Grados de participación comunitaria (\%s respecto al total)

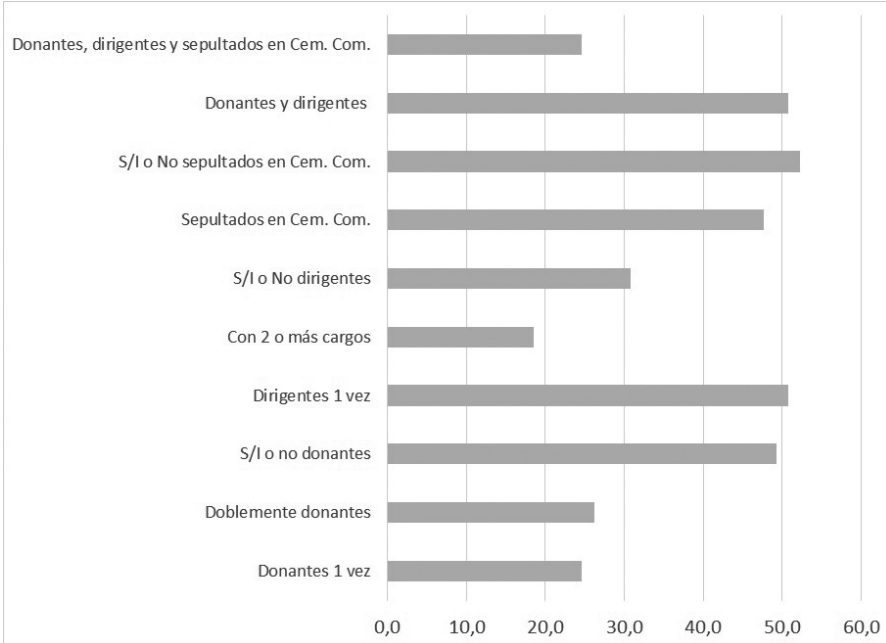

Una forma más evidente de mostrar un notable compromiso asociativo es identificando el porcentaje de la muestra que fue donante y, que además, ocupó algún cargo en las organizaciones comunitarias. En paralelo, también es posible medir el porcentaje de quienes -junto con lo anterior y según los registros disponibles- fueron sepultados con ritual judío. Esto podría catalogarse como grados de participación extrema y se expresan en el gráfico 12 .

GrÁFICO 12

Grados de participación comunitaria extrema

(\% respecto al total)

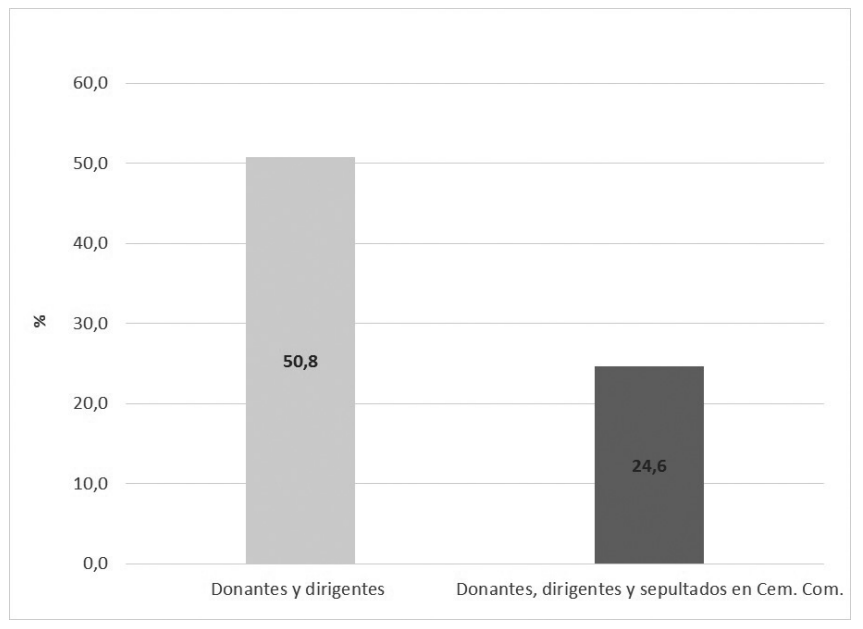


Aquí es posible apreciar que al menos un $50 \%$ de las sesenta y cinco personas estudiadas fueron donantes y dirigentes de organizaciones comunitarias. Al considerar una posible emigración a Santiago, el porcentaje de donantes, dirigentes y sepultados con ritual judío decae a alrededor de un $25 \%$, lo que no deja de evidenciar cuál habría sido el núcleo duro de la red asociativa judía en Valparaíso y Viña del Mar hasta 1944.

De tal modo, no debe sorprender que la directiva de la organización comunitaria judía que ya era más importante hacia 1944 en Valparaíso, la Sociedad Unión Israelita de Instrucción y Beneficencia de Valparaíso (SUIV) estuviera constituida por nueve personas, que a excepción de Israel Rosemblit, han aparecido varias veces a lo largo de este trabajo: presidente: Felipe Schapira; vicepresidentes: León Link y Samuel Michaely; tesorero: José Roscinsky; secretario: Mauricio Volonsky; vocales: Carlos Kusnier e Israel Rosemblit y revisores de cuentas: Julio Weinstein e Isidoro Froimovich ${ }^{21}$.

\section{CONCLUSIONES}

A partir de una rica base de datos sobre sesenta y cinco emprendedores de origen judío, que fueron primera generación en Chile y ejercieron su actividad económica en Valparaíso y Viña del Mar entre 1920 y 1944 es posible concluir lo siguiente.

Los establecimientos fundados por inmigrantes judíos directos en Valparaíso y Viña del Mar entre 1920 y 1944 estuvieron bastante diseminados en términos de propiedad. En otro ámbito, si bien la gran mayoría de ellos pertenecía al sector terciario, estos abarcaban otras actividades fuera del comercio (hospederías, farmacias, tintorerías, casa de cambio y servicios médicos); asimismo, existían otras actividades de orden manufacturero/fabril, que incluían a numerosos pequeños talleres y medianos e, incluso, a establecimientos propiamente industriales. A su vez, los establecimientos comerciales se concentraron en peleterías y fábricas de artículos de cuero, sastrerías, mueblerías y venta minoritaria de ropa y lencerías.

Es posible construir un relato aproximado de cómo fueron evolucionando estos emprendimientos a partir de la distribución de su escala de operaciones hacia 1944 y del año en que aparece el primer registro de cada establecimiento. Ellos sugieren un paulatino y gradual proceso de acumulación desde un modesto primer emprendimiento dedicado a la venta ambulante al detalle y a plazos para, luego, alquilar un local modesto. Si las condiciones mejoraban y se acumulaban nuevas experiencias y recursos, podían generarse un eslabonamiento hacia atrás, consistente en la creación de un pequeño taller en el mismo sitio, y ya en un cuarto momento, se podía pensar en establecer una tienda sucursal, comprar un inmueble de mayor tamaño y convertir la pequeña tienda en un establecimiento dedicado a las importaciones y a la venta al por mayor o, incluso, levantar un establecimiento industrial o ampliar el volumen de servicios (hospederías más grandes). Por cierto, cuando alguno de ellos tuvo estudios universitarios parciales o completos, pudo romper de algún modo esta lógica, ya que su base de acumulación era su grado de cualificación. En ese sentido, el momento en que se producía la inmigración

\footnotetext{
${ }^{21}$ Aguirre, op. cit., p. 14.
} 
y el nivel de educación con que se llegaba, podían ser muy influyentes en la forma en que cada uno se insertaba dentro de este ciclo general. En cualquier caso, la presencia mayoritaria de la pequeña tienda-taller hacia 1944 tiende a reforzar la idea de que la mayoría de estos emprendedores estaba entrando a una fase de consolidación en esa fecha.

La localización de estos establecimientos converge con la descripción de su gradual expansión, en la medida que, a diferencia del típico barrio comercial judío, muy bien delimitado dentro de un cuadrante -y que es posible de hallar en muchas ciudades del mundo en esta época- se desplegó a lo largo de los grandes ejes que comunicaban longitudinalmente a Valparaíso a través del Plan, tratando de satisfacer una variada demanda de artículos procedente de un heterogéneo mosaico social, que funcionaba como población flotante a lo largo del día.

En cuanto a los rasgos personales de estos emprendedores, que, si bien la gran mayoría de ellos fueron de origen askenazí, aquellos de origen sefardí lograron ostentar un protagonismo mucho mayor que el que les habría correspondido de manera proporcional según el tamaño relativo de cada grupo, situación que parece haberse acentuado en esas dos primeras décadas, en la medida que los grandes flujos de refugiados -donde el predominio askenazí era absoluto-comenzaron a arribar recién hacia 1939-1940.

En otro ámbito, la inmensa mayoría de estos inmigrantes no contaba con estudios universitarios - completos o incompletos- y los que de forma excepcional los tenían muestran aquella trayectoria que ya se ha comentado, y que se alejaba del relato general aplicable a los menos cualificados, aunque debe señalarse que es probable que esa misma mayoría haya contado con estudios primarios completos, y muchos de ellos, con estudios secundarios completos, una realidad muy por sobre la media educacional del país de acogida en esa época.

Así y todo, a estos emprendedores les tomó al menos una década consolidar sus actividades, situación que tal vez debe haber estado también asociada a los extendidos efectos de la Gran Depresión en Chile hasta 1939, momento en el que se recupera el PIB de 1929. Esta situación paralizó la inversión y consolidó la tendencia a interrumpir los procesos de expansión y debe haber mermado de forma severa la creación de nuevos establecimientos. El listado cronológico de establecimientos también corrobora la existencia de algunos encadenamientos verticales y horizontales, aparición de sucursales y aumento en la escala de operaciones.

Por otro lado, se recoge evidencia significativa en cuanto a que estos emprendedores fueron la base material sobre la que se asentó la vida asociativa judía en Valparaíso y Viña del Mar entre 1920 y 1944.

En efecto, un porcentaje aproximado al 50\% de las personas estudiadas donó al menos a una causa comunitaria -nacional o en Israel-, fue socio u ocupó algún cargo directivo en una organización comunitaria y fue sepultado con un ritual propiamente judío. Cruzando esas tres formas de compromiso comunitario, fue posible observar que una cifra muy similar cumplió las dos primeras categorías y un $25 \%$ del total materializó las tres, revelando niveles importantes de compromiso integral a su comunidad.

De ese modo, es posible afirmar que ha podido corroborarse que estos sesenta y cinco emprendedores sostuvieron económicamente el tejido asociativo temprano de la comunidad judía en Valparaíso y Viña del Mar hasta 1944. De hecho, este hecho, como ha 
ocurrido en muchas comunidades inmigrantes, posteriormente derivó en un relato épico dentro de la misma comunidad. Pero por desgracia, las nuevas dinámicas que se instauraron a partir de una segunda y tercera generación desde la década de 1950 hoy hacen ver este relato con un velo nostálgico, casi imposible de replicar. 